\title{
Search for third-generation scalar leptoquarks and heavy right-handed neutrinos in final states with two tau leptons and two jets in proton-proton collisions at $\sqrt{\mathrm{s}}=13 \mathrm{TeV}$
}

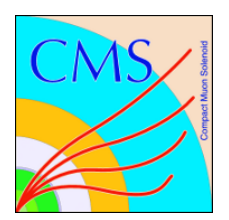

\section{The CMS collaboration}

E-mail: cms-publication-committee-chair@cern.ch

ABSTRACT: A search is performed for third-generation scalar leptoquarks and heavy righthanded neutrinos in events containing one electron or muon, one hadronically decaying $\tau$ lepton, and at least two jets, using a $\sqrt{s}=13 \mathrm{TeV}$ pp collision data sample corresponding to an integrated luminosity of $12.9 \mathrm{fb}^{-1}$ collected with the CMS detector at the LHC in 2016. The number of observed events is found to be in agreement with the standard model prediction. A limit is set at $95 \%$ confidence level on the product of the leptoquark pair production cross section and $\beta^{2}$, where $\beta$ is the branching fraction of leptoquark decay to a $\tau$ lepton and a bottom quark. Assuming $\beta=1$, third-generation leptoquarks with masses below $850 \mathrm{GeV}$ are excluded at $95 \%$ confidence level. An additional search based on the same event topology involves heavy right-handed neutrinos, $\mathrm{N}_{\mathrm{R}}$, and right-handed $\mathrm{W}$ bosons, $\mathrm{W}_{\mathrm{R}}$, arising in a left-right symmetric extension of the standard model. In this search, $\mathrm{W}_{\mathrm{R}}$ bosons are assumed to decay to a tau lepton and $\mathrm{N}_{\mathrm{R}}$ followed by the decay of the $\mathrm{N}_{\mathrm{R}}$ to a tau lepton and an off-shell $\mathrm{W}_{\mathrm{R}}$ boson. Assuming the mass of the right-handed neutrino to be half of the mass of the right-handed $\mathrm{W}$ boson, $\mathrm{W}_{\mathrm{R}}$ boson masses below $2.9 \mathrm{TeV}$ are excluded at $95 \%$ confidence level. These results improve on the limits from previous searches for third-generation leptoquarks and heavy right-handed neutrinos with $\tau$ leptons in the final state.

Keywords: Beyond Standard Model, Hadron-Hadron scattering (experiments)

ArXiv EPrint: 1703.03995 


\section{Contents}

1 Introduction 1

2 The CMS detector and Monte Carlo event samples 2

3 Event reconstruction and selection 3

4 Background estimation $\quad 5$

5 Systematic uncertainties $\quad 6$

6 Results $\quad 7$

$\begin{array}{lll}7 & \text { Summary } & 10\end{array}$

$\begin{array}{lr}\text { The CMS collaboration } & 16\end{array}$

\section{Introduction}

A number of extensions of the standard model (SM) have been proposed that predict an enhanced production rate for events containing pairs of quarks and pairs of third-generation leptons. One such theoretical proposal involves the existence of particles called leptoquarks (LQs), which carry color charge, fractional electric charge, and both lepton and baryon quantum numbers. The LQs arise in many models, including grand unified theories [1], compositeness models $[2,3]$, and superstring theories [4]. If LQs exist, they will decay into a lepton and a quark. At the CERN LHC, LQ pairs are predominantly produced via gluon-gluon fusion and quark-antiquark annihilation. Based on the latest experimental constraints reviewed in [5], we assume that contribution of t-channel production of LQ pairs involving Yukawa coupling of a LQ, a lepton, and a quark, is small and neglected in this analysis and the main free parameter is the mass of LQ. However, the branching fraction for the decay of a LQ into a quark and a charged lepton, $\beta$, depends on the details of the model under consideration. In this analysis we focus on the decay of a pair of third-generation LQs resulting in two $\tau$ leptons and two jets originating from $\mathrm{b}$ quark fragmentation.

A similar final state is expected in theories that postulate that the masses of the familiar left-handed neutrinos arise not from the Higgs field, but from a mechanism that involves the existence of right-handed neutrinos. One of the appealing features of left-right (L-R) symmetric extensions [6] of the SM is that these models predict the existence of new heavy charged $\left(\mathrm{W}_{\mathrm{R}}\right)$ and neutral $\left(\mathrm{Z}_{\mathrm{R}}\right)$ gauge bosons that could be produced at LHC 
energies. Heavy neutrinos $\left(\mathrm{N}_{\mathrm{e}}, \mathrm{N}_{\mu}, \mathrm{N}_{\tau}\right)$ naturally arise as the right-handed (RH) partners of the SM neutrinos in these L-R extensions through the see-saw mechanism [7].

In this paper, we search for these two processes by selecting final states containing two $\tau$ leptons and two jets originating from the hadronization of quarks. A search for pair production of third-generation scalar LQs is pursued by looking for events containing two $\tau$ leptons and two $\mathrm{b}$ quarks. We also search for the production of a $\mathrm{W}_{\mathrm{R}}$ boson from quarkantiquark annihilation. A heavy right-handed neutrino is produced from the decay of the $\mathrm{W}_{\mathrm{R}}$ boson following the decay chain $\mathrm{W}_{\mathrm{R}} \rightarrow \tau+\mathrm{N}_{\tau}$, where $\mathrm{N}_{\tau} \rightarrow \tau+\mathrm{W}_{\mathrm{R}}^{*} \rightarrow \tau+\mathrm{q} \overline{\mathrm{q}}$. In both searches, we focus on signatures with one of the $\tau$ leptons decaying into an electron or a muon, referred to as a leptonic decay $\tau_{\ell}$ in the following, and the other $\tau$ lepton decaying hadronically, denoted by $\tau_{\mathrm{h}}$.

Previous searches for third-generation LQs have been carried out at pp, $\mathrm{p} \overline{\mathrm{p}}, \mathrm{e}^{+} \mathrm{e}^{-}$, and ep colliders and the most recent results are given in $[8,9]$ and references therein. The most stringent lower limit on the mass of scalar third-generation LQs to date, based on the final state with two $\tau$ leptons and two b jets and assuming $\beta=1$, is $740 \mathrm{GeV}$ at $95 \%$ confidence level (CL), from the CMS experiment [10, 11]. Previous searches for heavy neutrinos have been performed at LEP [12, 13], excluding heavy neutrino masses below approximately $100 \mathrm{GeV}$. Further searches at LHC have been performed in the dielectron and dimuon channels and have excluded $\mathrm{W}_{\mathrm{R}}$ bosons with mass up to $3 \mathrm{TeV}$ using data taken at $7 \mathrm{TeV}$ [14] and at $8 \mathrm{TeV}$ [15]. Using $2.1 \mathrm{fb}^{-1}$ of data of $13 \mathrm{TeV}$ pp collisions collected in 2015, the CMS experiment searched for heavy neutrinos and right-handed charged bosons using events in which both $\tau$ leptons decay hadronically. That analysis excluded $\mathrm{W}_{\mathrm{R}}$ bosons with masses below $2.35(1.63) \mathrm{TeV}$ at $95 \% \mathrm{CL}$, assuming the $\mathrm{N}_{\tau}$ mass is $0.8(0.2)$ times the mass of $\mathrm{W}_{\mathrm{R}}$ boson [11]. In the present search, we use a $\sqrt{\mathrm{s}}=13 \mathrm{TeV}$ pp collision data sample corresponding to an integrated luminosity of $12.9 \mathrm{fb}^{-1}$ collected with the CMS detector in 2016.

\section{The CMS detector and Monte Carlo event samples}

The central feature of the CMS apparatus is a superconducting solenoid of $6 \mathrm{~m}$ internal diameter, providing a magnetic field of $3.8 \mathrm{~T}$. Within the solenoid volume are a silicon pixel and strip tracker, a lead tungstate crystal electromagnetic calorimeter (ECAL), and a brass and scintillator hadron calorimeter, each composed of a barrel and two endcap sections. Extensive forward calorimetry complements the coverage provided by the barrel and endcap detectors. Muons are detected in gas-ionisation detectors embedded in the steel flux-return yoke outside the solenoid. A detailed description of the CMS detector, together with a definition of the coordinate system used and the relevant kinematic variables, can be found in ref. [16].

The first level of the CMS triggering system, composed of custom hardware processors, uses information from the calorimeters and the muon detectors to select the most interesting events in a fixed time interval of less than $4 \mu \mathrm{s}$. The high-level trigger processor farm further decreases the event rate from around $100 \mathrm{kHz}$ to less than $1 \mathrm{kHz}$. 
Background and signal processes are modeled using the following simulated samples. The PYTHIA v8.205 generator [17] is used to model the signal and diboson (WW, WZ, and $\mathrm{ZZ)}$ processes. The LQ signal samples are generated with LQ masses ranging from 250 to $1500 \mathrm{GeV}$ in steps of $50 \mathrm{GeV}$. The branching fraction of the LQ to a $\tau$ lepton and a bottom quark is assumed to be $100 \%$. The signal samples are normalized to the next-to-next-toleading order $[18,19]$. The $\mathrm{W}_{\mathrm{R}}$ signal samples are generated with $\mathrm{W}_{\mathrm{R}}$ boson masses ranging from 1000 to $4000 \mathrm{GeV}$ in steps of $500 \mathrm{GeV}$ and the cross sections are computed in ref. [20]. The MadGraph v5.1.5 generator [21] is used to model $\mathrm{W}+$ jets and $\mathrm{Z}+$ jets processes. Single top production and t $\overline{\mathrm{t}}$ process are modelled with the POWHEG 2.0 [22-24] generator. The NNPDF 3.0 [25] Parton Distribution Functions (PDF) are used, and all simulated samples are interfaced with PYTHIA with the CUETP8M1 tune [26] to describe parton showering and hadronization. Additional inelastic pp interactions (pileup) generated by PYTHIA are overlaid on all simulated events, according to the luminosity profile of the analyzed data. All the generated signal and background samples are processed with the simulation of the CMS detector based on Geant4 [27]. Small differences between data and simulation in trigger, in particle identification and isolation efficiencies, and in the resolution of the $p_{\mathrm{T}}$ of jets and missing transverse momentum are corrected by applying scale factors to simulated events, as detailed below.

\section{Event reconstruction and selection}

The particle-flow $(\mathrm{PF})$ algorithm [28, 29], which exploits information from all subdetectors, is used to identify individual particles, such as charged and neutral hadrons, muons, electrons, and photons. These reconstructed particles are used as input for reconstructing more complex objects such as $\tau_{\mathrm{h}}$ candidates, jets, and variables like missing transverse momentum.

The reconstructed interaction vertex with the largest value of $\sum_{i}\left(p_{\mathrm{T}}^{i}\right)^{2}$, where $p_{\mathrm{T}}^{i}$ is the transverse momentum of the $i$ th track associated with the vertex, is selected as the primary vertex of the event. This vertex is used as the reference vertex for all the objects reconstructed using the $\mathrm{PF}$ algorithm.

Electrons are reconstructed by matching the energy deposits in the ECAL to tracks reconstructed in the silicon pixel and strip detectors. The electrons selected in this analysis are required to have transverse momenta $p_{\mathrm{T}}>50 \mathrm{GeV}$ and pseudorapidity $|\eta|<2.1$ [30]. The identification and isolation of electrons are based on a multivariate technique [31] and selected electrons must satisfy tight electron identification and isolation criteria.

Muon reconstruction starts by matching tracks in the silicon tracker with tracks in the outer muon spectrometer [32]. A global muon track is fitted to the hits from both tracks. Muons are required to have $p_{\mathrm{T}}>50 \mathrm{GeV}$ and $|\eta|<2.1$. Quality selection criteria are applied to the muon tracks to distinguish muons originating from particle collisions with those muons coming from cosmic rays. In addition, muons are required to pass isolation criteria to separate prompt muons from those associated with a jet, usually from the semileptonic decays of heavy quarks. 
The hadron-plus-strips algorithm $[33,34]$ is used to reconstruct $\tau_{\mathrm{h}}$ candidates. It starts from a jet and searches for candidates produced by the main hadronic decay modes of a $\tau$ lepton: either directly to one charged hadron, or via intermediate $\rho(770)$ and $\mathrm{a}_{1}(1280)$ mesons to one charged hadron plus one or two neutral pions, or three charged hadrons. The reconstructed $\tau_{\mathrm{h}}$ is required to have $|\eta|<2.3$ and $p_{\mathrm{T}}>50\left(p_{\mathrm{T}}>60\right) \mathrm{GeV}$ in the LQ (heavy $\mathrm{RH}$ neutrino) search. Hadronic tau lepton decays are identified by a multivariate technique that uses as inputs the isolation of the $\tau_{\mathrm{h}}$ and variables that are sensitive to its lifetime. A selection criterion is used that has an efficiency of approximately $65 \%$ for identifying hadronically decaying tau leptons and a probability of less than $1 \%$ for misidentifying jets as hadronic tau decays. Additional criteria are applied to remove electrons and muons reconstructed as $\tau_{\mathrm{h}}$ candidates.

The identified electron or muon and the $\tau_{\mathrm{h}}$ are required to originate from the same vertex and be spatially separated by $\Delta R \equiv \sqrt{\Delta \phi^{2}+\Delta \eta^{2}}>0.5$. To suppress background events such as diboson and $\mathrm{Z}+$ jets with bosons decay giving a final state with a pair of leptons, events containing additional electron or muon candidates with $p_{\mathrm{T}}>15 \mathrm{GeV}$, and which pass loose identification and isolation criteria, are rejected.

Jets are reconstructed using the anti- $k_{\mathrm{T}}$ algorithm with a distance parameter of $R=$ $0.4[35,36]$ using PF candidates. The jet energy is corrected for the average contribution from particles from other proton-proton collisions in the same or neighbouring bunch crossings (pileup) [37]. Additional corrections are applied to better reflect the true total momentum of the particles in the jet [38]. Selected jets are required to be within $|\eta|<2.4$ and have $p_{\mathrm{T}}>50 \mathrm{GeV}$, and to be separated from the selected electron or muon and the $\tau_{\mathrm{h}}$ by $\Delta R>0.5$. Further identification requirements are applied to distinguish genuine jets from those coming from pileup [39].

The transverse momentum imbalance, $\left(\vec{p}_{\mathrm{T}}^{\text {miss }}\right)$, is calculated as the negative vectorial sum of transverse momenta of all PF candidates, and corrected by propagating the corrections applied to identified jets [40]. A correction is applied to account for the effect of additional pileup interactions. In addition, several filters are employed to veto events with large $\vec{p}_{\mathrm{T}}^{\text {miss }}$ caused by detector effects.

Candidate events were collected using a set of triggers requiring the presence of either an electron or a muon candidate with $p_{\mathrm{T}}>45 \mathrm{GeV}$.

The search for LQs is based on a sample of events containing one light lepton, one $\tau_{\mathrm{h}}$ candidate, and at least two jets. At least one of the two leading jets is identified as originating from $\mathrm{b}$ quark hadronization (b-tagged) using the combined secondary vertex algorithm [41]. The chosen $b$ tagging working point corresponds to an identification efficiency of approximately $70 \%$ with about $1 \%$ misidentification rate from light quarks. The lepton and $\tau_{\mathrm{h}}$ candidate are required to have opposite electric charge. There are two possible combinations of two tau candidates, with two jets, and the combination that minimises the difference in masses between the two resulting tau candidate-jet systems is chosen. Additionally, the invariant mass of the system formed by the visible particles of the $\tau_{\mathrm{h}}$ candidate and a jet is required to be greater than $250 \mathrm{GeV}$.

The search for a $\mathrm{W}_{\mathrm{R}}$ boson decaying into a heavy neutrino uses the same data sample as used by the LQ search. The $\vec{p}_{\mathrm{T}}^{\text {miss }}$ is required to be above $50 \mathrm{GeV}$ and the invariant mass of the light lepton and the $\tau_{\mathrm{h}}$ is required to be greater than $150 \mathrm{GeV}$. 
In the LQ analysis, the fraction of signal events passing all selection cuts ranges between 1 and $5 \%$ for masses between 300 and $1500 \mathrm{GeV}$, and in the $\mathrm{W}_{\mathrm{R}}$ analysis the fraction varies between 2 and $7 \%$ for masses between 1 and $4 \mathrm{TeV}$.

The presence of a signal is investigated by analysing the distribution of $S_{\mathrm{T}}$. This is defined as the scalar sum of the $p_{\mathrm{T}}$ of the electron or muon, the $\tau_{\mathrm{h}}$ candidate, the two jets, and the missing transverse energy.

\section{Background estimation}

Several SM processes can mimic the signatures explored in this search. Production of $\mathrm{t} \overline{\mathrm{t}}$ pairs is the dominant background because of the presence of genuine leptons, $\vec{p}_{\mathrm{T}}^{\mathrm{miss}}$, and both light- and heavy-flavour jets. Additionally, the production of a $\mathrm{W}$ or $\mathrm{Z}$ boson in association with jets, production of a diboson or a single top quark, and Quantum ChromoDynamics (QCD) multijet processes can also contribute to the SM background contributions.

Simulated $\mathrm{t} \overline{\mathrm{t}}$ events are reweighted according to the top quark $p_{\mathrm{T}}$ distribution measured

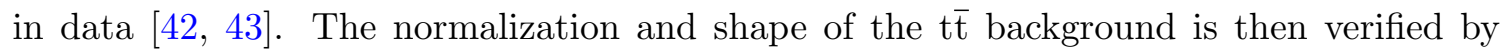
comparing to a data sample that consists of events containing an electron, a muon, and at least two jets and including all final selection requirements. The purity of t $\bar{t}$ events in this sample exceeds $95 \%$. Signal contamination in this control region is found to be negligible and does not affect the comparison of data with simulation even in the tail of

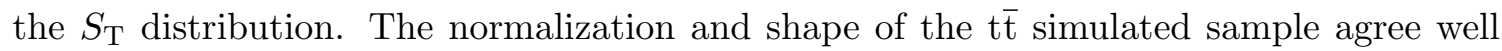
with those observed in data. Thus, the simulation is used to predict the te background in the signal region.

The $\mathrm{W}+$ jets background arises mainly from events with a genuine electron or muon originating from the leptonic decay of a $\mathrm{W}$ boson and an initial- or final-state radiation jet misidentified as a $\tau_{\mathrm{h}}$ candidate. The normalization and shape of the $\mathrm{W}$ background are obtained from simulation and a correction factor is applied to the normalization to take into account differences between data and simulation. The $\mathrm{W}$ background correction factor is estimated in a data sample that consists of $\mathrm{W} \rightarrow \mu \nu$ events with three or more jets. One of the jets is required to pass the $\tau_{\mathrm{h}}$ identification criteria. To reduce the contamination from $t \bar{t}$ background, events containing jets that pass the $b$ tagging criteria are rejected. The expected signal contamination in this sample is negligible. A binned maximum likelihood fit to the transverse mass distribution of the muon and $\vec{p}_{\mathrm{T}}^{\text {miss }}$ is then performed to derive the $\mathrm{W}$ background normalization correction factor. The transverse mass distribution is found to have the most discriminating power for separating the $\mathrm{W}$ background from the other backgrounds. As an input to the fit, the normalization and shape of all other contributions are estimated from simulation. The uncertainties in the cross sections of all backgrounds are included as nuisance parameters in the fit. The contamination from QCD multijet events is small and derived from simulation. The best fit value for the $\mathrm{W}$ normalization correction factor is found to be $1.0 \pm 0.2$, with the uncertainty including both statistical and systematic components. 
A similar procedure is repeated for the $\mathrm{e} \tau_{\mathrm{h}}$ channel in a control region containing events with an electron and three or more jets. The $\mathrm{W}+$ jets normalization factor measured in $\mathrm{W} \rightarrow \mathrm{e} \nu$ events is found to be consistent with the normalization factor derived in $\mathrm{W} \rightarrow \mu \nu$ events, albeit with a slightly larger uncertainty.

The contribution of the QCD multijet background to the signal region in both the $\mu \tau_{\mathrm{h}}$ and $\mathrm{e} \tau_{\mathrm{h}}$ channels is estimated from data. Events in the multijet control region are selected by inverting the $\tau_{\mathrm{h}}$ identification criteria: the $\tau_{\mathrm{h}}$ candidate is required to pass looser, but to fail tighter, identification criteria. The events are weighted by the $p_{\mathrm{T}}$-dependent probability for a jet satisfying loose isolation criteria to pass the tight $\tau_{\mathrm{h}}$ isolation criteria. This probability is measured as a function of jet $p_{\mathrm{T}}$ for $\mathrm{e} \tau_{\mathrm{h}}$ and $\mu \tau_{\mathrm{h}}$ channels separately, in independent data samples that are composed of events in which the lepton fails the isolation criteria and has the same charge as $\tau_{\mathrm{h}}$ candidate. This probability varies from $20 \%$ for a jet $p_{\mathrm{T}}$ of $50 \mathrm{GeV}$ to $2 \%$ for a jet $p_{\mathrm{T}}$ of $400 \mathrm{GeV}$ and is similar for both final states.

In addition to the principal backgrounds, which are estimated as discussed above, other minor backgrounds, arising from single top quark, Z boson, and diboson production, are estimated from simulation. The relative contributions of all these backgrounds are given in tables 1 and 2 , in section 6 . In these tables, the electroweak background represents the sum of the backgrounds from $\mathrm{W}+$ jets, $\mathrm{Z}$ boson, and diboson production. Additionally, for each channel the background estimation is compared with the observed data and with an estimated representative signal.

\section{$5 \quad$ Systematic uncertainties}

The results of the analysis are obtained from a binned fit to the $S_{\mathrm{T}}$ distributions in the e $\tau_{\mathrm{h}}$ and $\mu \tau_{\mathrm{h}}$ channels. Systematic uncertainties may affect the normalization and/or the shape of the $S_{\mathrm{T}}$ distribution of the signal and background processes.

The uncertainty in the integrated luminosity of the analysed dataset amounts to $6.2 \%$ [44]. Uncertainties in the muon and electron identification and trigger efficiency are determined using the "tag-and-probe" technique [45] and amount to $2 \%$ for identification and $5 \%$ for trigger efficiencies. The $\tau_{\mathrm{h}}$ identification efficiency $[33,46]$ is measured in bins of $\tau_{\mathrm{h}}$ candidate $p_{\mathrm{T}}$ in $\mathrm{Z} \rightarrow \tau \tau$ and $\mathrm{W} \rightarrow \tau \nu$ events and fitted by a linear function within the range 20 to $200 \mathrm{GeV}$. The uncertainty in the $\tau_{\mathrm{h}}$ identification efficiency measurement is $6 \%$ for $\tau$ leptons from the decay of $\mathrm{Z}$ bosons. The extrapolation to higher transverse momenta is taken into account by adding an uncertainty that increases linearly with $p_{\mathrm{T}}$ and has a value of $20 \%$ for a $p_{\mathrm{T}}$ of $200 \mathrm{GeV}$. This uncertainty has a direct effect on the $S_{\mathrm{T}}$ distribution and hence is considered as a shape uncertainty. Changes in the acceptance due to the uncertainty in the b tagging efficiency and in the mistag rate are measured to be between 3 and $5 \%$, depending on the process. The uncertainty in the normalization of the $t \overline{\mathrm{t}}$ background due to the PDF and scale uncertainties amounts to 5\% [47, 48]. A 10\% uncertainty is attributed to the $\mathrm{Z}$ boson background estimate, while the uncertainty in both the diboson and single top background estimates amounts to $15 \%$ [49]. The uncertainty in the yield of QCD multijet and W+jet backgrounds amounts to $30 \%$. The uncertainty in the signal acceptance due to the choice of the PDF set in the simulated sample is evaluated 
in accordance to the PDF4LHC recommendations [48, 50], by comparing the results obtained using the CTEQ6.6L, MSTW08, and NNPDF10 PDF sets [51-53] with those from the default PDF set (CTEQ6L1). This uncertainty amounts to 5\% [11].

The energy scales (ES) of the $\tau_{\mathrm{h}}$ candidate and the associated jet affect the shape of the $S_{\mathrm{T}}$ distribution and normalization of the signal and background processes. The effects of ES uncertainties on the analysis are estimated by varying the $\tau_{\mathrm{h}}$ and jet energies within their respective uncertainties and recomputing $S_{\mathrm{T}}$ after the final selection. The uncertainty in the $\tau_{\mathrm{h}}$ ES amounts to 3\% [33]. The uncertainty in the jet ES affects the $p_{\mathrm{T}}$ spectrum of the jets and consequently $\vec{p}_{\mathrm{T}}^{\text {miss }}$, and is applied to signal and all backgrounds that are estimated with MC simulation [54]. The uncertainties in the electron, muon, and $\vec{p}_{\mathrm{T}}^{\text {miss }}$ ES have a negligible effect on the $S_{\mathrm{T}}$ distribution. The uncertainty in the top quark $p_{\mathrm{T}}$ reweighting correction is derived by changing the event weight between zero and twice the nominal reweighting correction value $[42,43]$. All these three uncertainties are treated as correlated between the $\mathrm{e} \tau_{\mathrm{h}}$ and $\mu \tau_{\mathrm{h}}$ channels.

Finally, the effects of statistical uncertainties associated with the signal and background shapes or with the numbers of events in the data control regions are included in the analysis. The statistical uncertainties are uncorrelated across the bins in each background distribution [55].

Systematic and statistical uncertainties are represented by nuisance parameters in the fit. A log-normal probability distribution function is assumed for the nuisance parameters that affect the event yields of the various background contributions. Systematic uncertainties affecting the $S_{\mathrm{T}}$ distributions are assumed to have a Gaussian probability distribution function. Among those uncertainties, the $\tau_{\mathrm{h}} \mathrm{ES}$ and high $p_{\mathrm{T}} \tau_{\mathrm{h}}$ extrapolation uncertainties are uncorrelated between the $\mathrm{e} \tau_{\mathrm{h}}$ and $\mu \tau_{\mathrm{h}}$ channels, because of the different $\tau_{\mathrm{h}}$ identification criteria used to reduce the electron and muon mis-identification rate in each channel. The jet ES is treated as correlated across the two channels.

\section{Results}

A binned maximum likelihood fit to the $S_{\mathrm{T}}$ distribution has been applied to the e $\tau_{\mathrm{h}}$ and $\mu \tau_{\mathrm{h}}$ channels simultaneously. The signal production rate is constrained to the same value in the two channels. The $S_{\mathrm{T}}$ distributions for both the $\mathrm{LQ}$ and $\mathrm{W}_{\mathrm{R}}$ analyses are shown in figure 1. Shape, normalization and uncertainty are shown for the values of nuisances parameters obtained from the fit. No excess is seen above the SM expectation within the statistical and systematic uncertainties in both searches. The event yields observed in the leptoquark analysis and in the heavy right-handed W boson analysis are shown in tables 1 and 2, respectively, and compared to background expectations and to the estimated event yields for representative signals.

Upper limits on the product of the cross section and branching fractions are set at $95 \%$ CL using a modified frequentist criterion $\mathrm{CL}_{\mathrm{s}}[56,57]$, based on the binned distribution of the $S_{\mathrm{T}}$ variable. Figure 2 (left) shows the observed and expected $95 \%$ CL upper limit on the product of cross section and branching fraction in the LQ analysis. The observed (expected) 95\% CL mass limit for third-generation scalar LQ is determined to be 850 

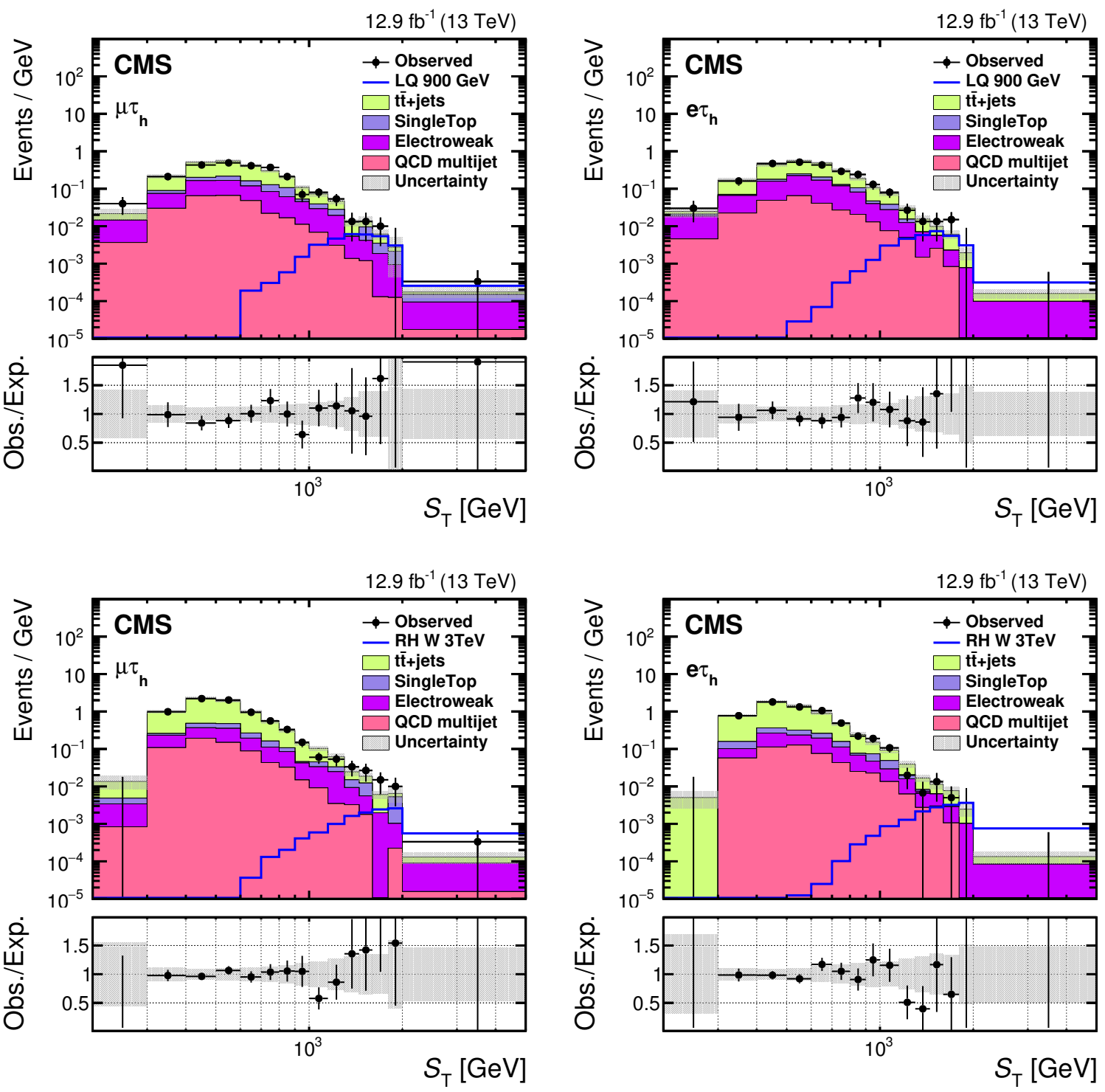

Figure 1. Measured $S_{\mathrm{T}}$ distribution in the $\mu \tau_{\mathrm{h}}$ (left) and e $\tau_{\mathrm{h}}$ (right) channels of the LQ (upper) and heavy RH neutrino (lower) analyses, compared to the expected SM background contribution. A hypothetical LQ signal of mass $M_{\mathrm{LQ}}=900 \mathrm{GeV}$ and a hypothetical heavy $\mathrm{W}_{\mathrm{R}}$ signal of mass $M_{\mathrm{W}_{\mathrm{R}}}=3 \mathrm{TeV}$ are overlaid to illustrate the sensitivity. The electroweak background represents the sum of $\mathrm{W}$ boson, $\mathrm{Z}$ boson, and diboson production. The last bin of each plot contains overflow events. A binned maximum likelihood fit is performed on the $S_{\mathrm{T}}$ distribution. The uncertainty bands represent the sum in quadrature of statistical and systematic uncertainties, obtained from the fit. The lower panels in all plots compare the observed and expected events in each bin. 


\begin{tabular}{|l|cc|}
\hline Process & $\mathrm{e} \tau_{\mathrm{h}}$ & $\mu \tau_{\mathrm{h}}$ \\
\hline $\mathrm{t} \overline{\mathrm{t}}+$ jets & $136.8 \pm 13.2$ & $145.6 \pm 14.6$ \\
SingleTop & $15.7 \pm 3.1$ & $27.6 \pm 4.5$ \\
Electroweak & $69.6 \pm 10.5$ & $53.3 \pm 9.0$ \\
QCD multijet & $25.0 \pm 6.8$ & $28.9 \pm 7.4$ \\
\hline Total expected background & $247.1 \pm 16.8$ & $255.4 \pm 16.1$ \\
\hline LQ 900 GeV & $6.0 \pm 0.3$ & $5.7 \pm 0.2$ \\
\hline Observed data & 249 & 250 \\
\hline
\end{tabular}

Table 1. Number of events observed in the $\mathrm{e} \tau_{\mathrm{h}}$ and $\mu \tau_{\mathrm{h}}$ channels of the LQ analysis compared to the background expectations and to the event yield expected for a representative LQ signal of mass $900 \mathrm{GeV}$. The quoted uncertainties represent the sum of statistical and systematic uncertainties and are obtained by the binned maximum likelihood fit of the distribution in $S_{\mathrm{T}}$, as described in the text.

\begin{tabular}{|l|cc|}
\hline Process & $\mathrm{e} \tau_{\mathrm{h}}$ & $\mu \tau_{\mathrm{h}}$ \\
\hline $\mathrm{t} \overline{\mathrm{t}}+$ jets & $456.2 \pm 25.8$ & $557.6 \pm 30.7$ \\
SingleTop & $41.6 \pm 7.1$ & $47.6 \pm 8.1$ \\
Electroweak & $60.2 \pm 26.8$ & $83.6 \pm 33.2$ \\
QCD multijet & $48.6 \pm 13.1$ & $65.7 \pm 16.8$ \\
\hline Total expected background & $606.6 \pm 33.5$ & $754.5 \pm 38.7$ \\
\hline RHW 3000 GeV & $4.8 \pm 0.3$ & $3.5 \pm 0.3$ \\
\hline Observed data & 606 & 751 \\
\hline
\end{tabular}

Table 2. Number of events observed in the $\mathrm{e} \tau_{\mathrm{h}}$ and $\mu \tau_{\mathrm{h}}$ channels of the heavy right-handed $\mathrm{W}$ analysis compared to the background expectations and to the event yield expected for a representative right-handed $\mathrm{W}$ boson signal of mass $3 \mathrm{TeV}$. The quoted uncertainties represent the sum of statistical and systematic uncertainties and are obtained by the binned maximum likelihood fit of the distribution in $S_{\mathrm{T}}$, as described in the text.

(900) $\mathrm{GeV}$, respectively, assuming $\beta=1$, namely a $100 \%$ branching fraction for the LQ to decay into a $\tau$ lepton and a bottom quark. Figure 2 (right) shows the 95\% CL observed and expected exclusion limits on the LQ mass, as a function of $\beta$.

Figure 3 (left) shows the observed and expected upper limits at 95\% CL on the product of cross section and branching fraction for the $\mathrm{W}_{\mathrm{R}} \rightarrow \tau \mathrm{N}_{\tau}$ analysis. Assuming the mass of the neutrino to be half the mass of the $\mathrm{W}_{\mathrm{R}}$ boson, the observed (expected) limit at 95\% CL on the mass of heavy right-handed $\mathrm{W}_{\mathrm{R}}$ bosons is determined to be 2.9 (3.0) TeV, respectively. Figure 3 (right) shows the observed and expected 95\% CL upper limits on the production cross section as functions of $M_{\mathrm{W}_{\mathrm{R}}}$ and $M_{\mathrm{N}_{\tau}}$. The blue curve in the left plot represent the theoretical production cross section of $\mathrm{W}_{\mathrm{R}}$ boson times branching fraction of the $\mathrm{W}_{\mathrm{R}}$ boson to a $\tau$ lepton and $\mathrm{RH}$ neutrino, assuming mass of $\mathrm{RH}$ neutrino to be half the mass of $\mathrm{W}_{\mathrm{R}}$ boson. 

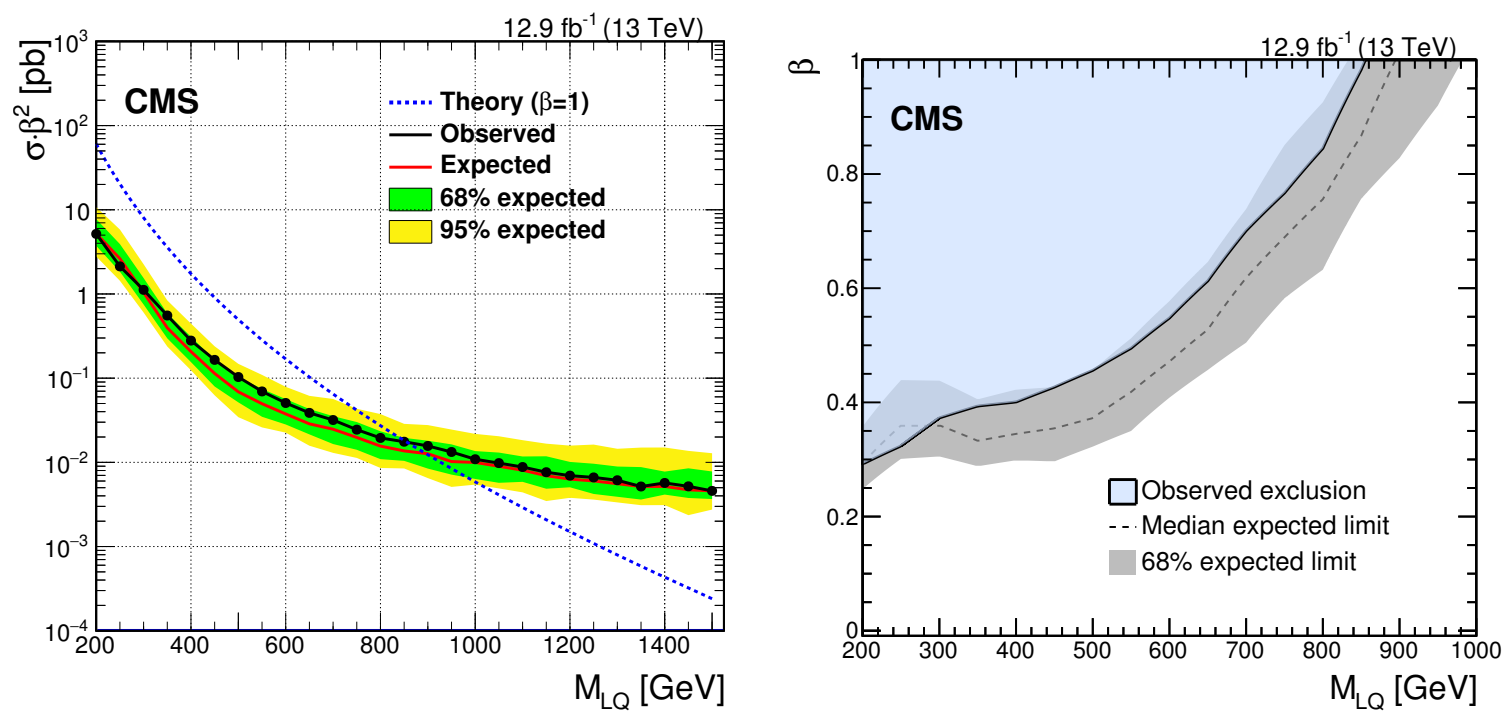

Figure 2. Observed and expected limits at 95\% CL on the product of cross section and branching fraction squared, obtained from the combination of the $\mathrm{e} \tau_{\mathrm{h}}$ and $\mu \tau_{\mathrm{h}}$ channels, in the LQ analysis (left) and 95\% CL observed and expected exclusion limits on the LQ mass, as a function of $\beta$ (right). In the left plot, the green and yellow bands represent the one and two standard deviation uncertainties in the expected limits. The dashed dark blue curve represents the theoretical LQ pair production cross section, assuming $\beta=100 \%[18,19]$. In the right plot, the grey band represents the one standard deviation uncertainty in the expected limit.

\section{Summary}

Searches have been performed for third-generation scalar leptoquarks and for heavy righthanded neutrinos in events containing one electron or muon, one hadronically decaying $\tau$ lepton, and two or more jets, using pp collision data at $\sqrt{s}=13 \mathrm{TeV}$, recorded by the CMS detector at the LHC and corresponding to an integrated luminosity of $12.9 \mathrm{fb}^{-1}$. The data are found to be in good agreement with the standard model prediction in both analyses. A limit at $95 \%$ confidence level is set on the product of the leptoquark pair production cross section and $\beta^{2}$, where $\beta$ denotes the branching fraction for the decay of the leptoquark into a $\tau$ lepton and a bottom quark. Assuming $\beta=1$, third-generation leptoquarks with masses below $850 \mathrm{GeV}$ are excluded at $95 \%$ confidence level. In the heavy $\mathrm{RH}$ neutrino analysis, considering the decay $\mathrm{W}_{\mathrm{R}} \rightarrow \tau \mathrm{N}_{\mathrm{R}}$ and assuming the mass of the heavy neutrino to be half the mass of the $\mathrm{W}_{\mathrm{R}}$ boson, we exclude $\mathrm{W}_{\mathrm{R}}$ boson masses below $2.9 \mathrm{TeV}$ at $95 \%$ confidence level. These are the best mass limits to date for third-generation leptoquarks and heavy right-handed neutrinos with $\tau$ leptons in the final state.

\section{Acknowledgments}

We congratulate our colleagues in the CERN accelerator departments for the excellent performance of the LHC and thank the technical and administrative staffs at CERN and 

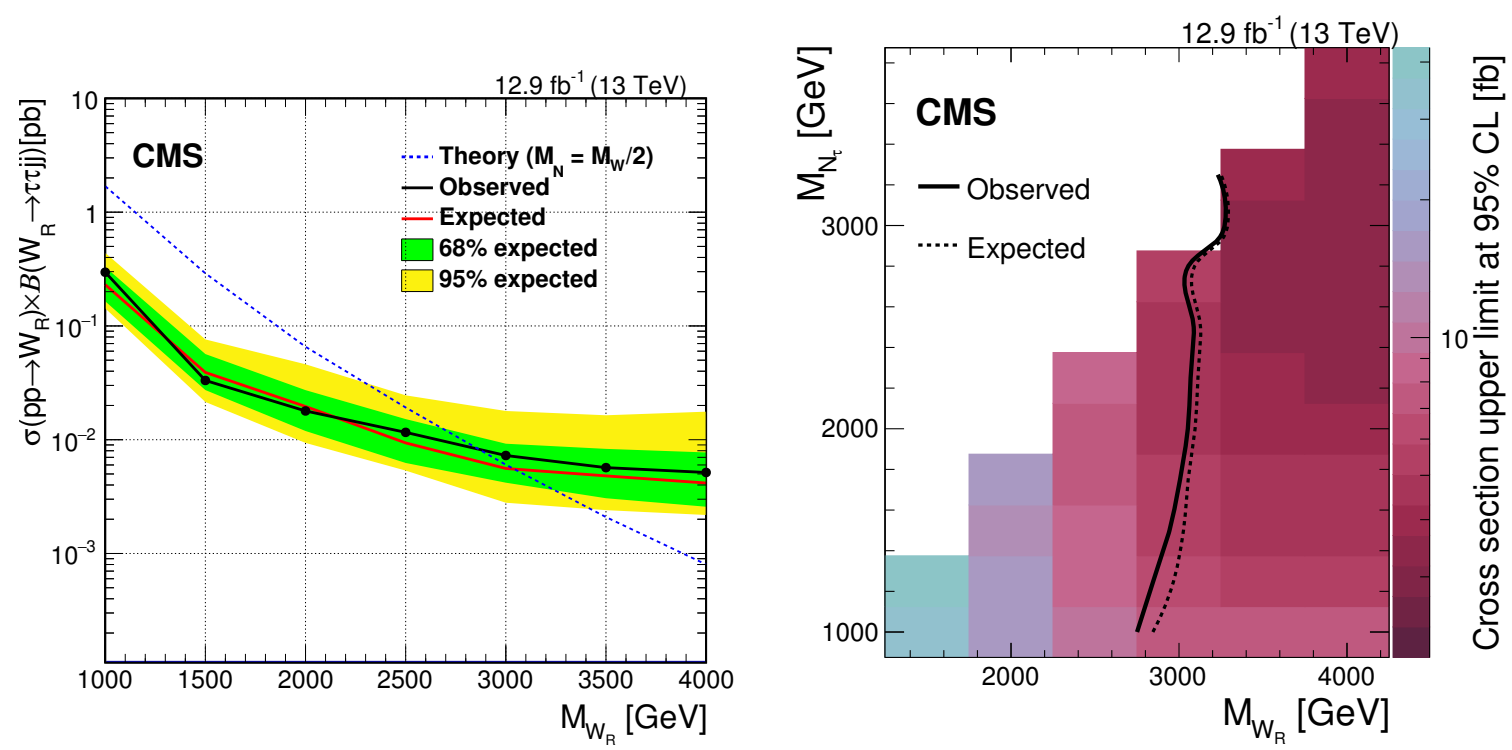

Figure 3. Observed and expected limits at 95\% CL on the product of cross section and branching fraction, obtained from the combination of the $\mathrm{e} \tau_{\mathrm{h}}$ and $\mu \tau_{\mathrm{h}}$ channels in the heavy right-handed neutrino analysis (left) and the observed and expected limits at 95\% CL on the production cross section as a function of $\mathrm{M}_{\mathrm{W}_{R}}$ and $\mathrm{M}_{\mathrm{N}_{\mathrm{R}}}$ (right). The green and yellow bands represent the one and two standard deviation uncertainties in the expected limits. The dashed dark blue curve represents the theoretical prediction for the product of the $\mathrm{W}_{\mathrm{R}}$ boson production cross section and the branching fraction for decay to a $\tau$ lepton and $\mathrm{RH}$ neutrino, assuming the mass of the $\mathrm{RH}$ neutrino to be half the mass of the $\mathrm{W}_{\mathrm{R}}$ boson [20].

at other CMS institutes for their contributions to the success of the CMS effort. In addition, we gratefully acknowledge the computing centres and personnel of the Worldwide LHC Computing Grid for delivering so effectively the computing infrastructure essential to our analyses. Finally, we acknowledge the enduring support for the construction and operation of the LHC and the CMS detector provided by the following funding agencies: BMWFW and FWF (Austria); FNRS and FWO (Belgium); CNPq, CAPES, FAPERJ, and FAPESP (Brazil); MES (Bulgaria); CERN; CAS, MoST, and NSFC (China); COLCIENCIAS (Colombia); MSES and CSF (Croatia); RPF (Cyprus); SENESCYT (Ecuador); MoER, ERC IUT, and ERDF (Estonia); Academy of Finland, MEC, and HIP (Finland); CEA and CNRS/IN2P3 (France); BMBF, DFG, and HGF (Germany); GSRT (Greece); OTKA and NIH (Hungary); DAE and DST (India); IPM (Iran); SFI (Ireland); INFN (Italy); MSIP and NRF (Republic of Korea); LAS (Lithuania); MOE and UM (Malaysia); BUAP, CINVESTAV, CONACYT, LNS, SEP, and UASLP-FAI (Mexico); MBIE (New Zealand); PAEC (Pakistan); MSHE and NSC (Poland); FCT (Portugal); JINR (Dubna); MON, RosAtom, RAS, RFBR and RAEP (Russia); MESTD (Serbia); SEIDI, CPAN, PCTI and FEDER (Spain); Swiss Funding Agencies (Switzerland); MST (Taipei); ThEPCenter, IPST, STAR, and NSTDA (Thailand); TUBITAK and TAEK (Turkey); NASU and SFFR (Ukraine); STFC (United Kingdom); DOE and NSF (U.S.A.).

Individuals have received support from the Marie-Curie programme and the European Research Council and EPLANET (European Union); the Leventis Foundation; the 
A.P. Sloan Foundation; the Alexander von Humboldt Foundation; the Belgian Federal Science Policy Office; the Fonds pour la Formation à la Recherche dans l'Industrie et dans l'Agriculture (FRIA-Belgium); the Agentschap voor Innovatie door Wetenschap en Technologie (IWT-Belgium); the Ministry of Education, Youth and Sports (MEYS) of the Czech Republic; the Council of Science and Industrial Research, India; the HOMING PLUS programme of the Foundation for Polish Science, cofinanced from European Union, Regional Development Fund, the Mobility Plus programme of the Ministry of Science and Higher Education, the National Science Center (Poland), contracts Harmonia 2014/14/M/ST2/00428, Opus 2014/13/B/ST2/02543, 2014/15/B/ST2/03998, and 2015/19/B/ST2/02861, Sonata-bis 2012/07/E/ST2/01406; the National Priorities Research Program by Qatar National Research Fund; the Programa Clarín-COFUND del Principado de Asturias; the Thalis and Aristeia programmes cofinanced by EU-ESF and the Greek NSRF; the Rachadapisek Sompot Fund for Postdoctoral Fellowship, Chulalongkorn University and the Chulalongkorn Academic into Its 2nd Century Project Advancement Project (Thailand); and the Welch Foundation, contract C-1845.

Open Access. This article is distributed under the terms of the Creative Commons Attribution License (CC-BY 4.0), which permits any use, distribution and reproduction in any medium, provided the original author(s) and source are credited.

\section{References}

[1] H. Georgi and S.L. Glashow, Unity of All Elementary Particle Forces, Phys. Rev. Lett. 32 (1974) 438 [INSPIRE].

[2] B. Schrempp and F. Schrempp, Light leptoquarks, Phys. Lett. B 153 (1985) 101 [INSPIRE].

[3] W. Buchmüller, R. Rückl and D. Wyler, Leptoquarks in lepton-quark collisions, Phys. Lett. B 191 (1987) 442 [Erratum ibid. B 448 (1999) 320] [INSPIRE].

[4] J.L. Hewett and T.G. Rizzo, Low-Energy Phenomenology of Superstring Inspired $E_{6}$ Models, Phys. Rept. 183 (1989) 193 [INSPIRE].

[5] I. Doršner, S. Fajfer, A. Greljo, J.F. Kamenik and N. Košnik, Physics of leptoquarks in precision experiments and at particle colliders, Phys. Rept. 641 (2016) 1 [arXiv:1603.04993] [INSPIRE].

[6] R.N. Mohapatra and J.C. Pati, A Natural Left-Right Symmetry, Phys. Rev. D 11 (1975) 2558 [INSPIRE].

[7] W.-Y. Keung and G. Senjanović, Majorana Neutrinos and the Production of the Right-handed Charged Gauge Boson, Phys. Rev. Lett. 50 (1983) 1427 [INSPIRE].

[8] ATLAS collaboration, Search for third generation scalar leptoquarks in pp collisions at $\sqrt{s}=7 \mathrm{TeV}$ with the ATLAS detector, JHEP 06 (2013) 033 [arXiv:1303.0526] [inSPIRE].

[9] CMS collaboration, Search for pair production of third-generation scalar leptoquarks and top squarks in proton-proton collisions at $\sqrt{s}=8 \mathrm{TeV}$, Phys. Lett. B 739 (2014) 229 [arXiv:1408.0806] [INSPIRE]. 
[10] CMS collaboration, Search for pair production of third-generation leptoquarks and top squarks in pp collisions at $\sqrt{s}=7$ TeV, Phys. Rev. Lett. 110 (2013) 081801 [arXiv:1210.5629] [INSPIRE].

[11] CMS collaboration, Search for heavy neutrinos or third-generation leptoquarks in final states with two hadronically decaying $\tau$ leptons and two jets in proton-proton collisions at $\sqrt{s}=13 \mathrm{TeV}$, JHEP 03 (2017) 077 [arXiv: 1612.01190] [INSPIRE].

[12] DELPHI collaboration, P. Abreu et al., Search for neutral heavy leptons produced in $Z$ decays, Z. Phys. C 74 (1997) 57 [Erratum ibid. C 75 (1997) 580] [InSPIRE].

[13] L3 collaboration, O. Adriani et al., Search for isosinglet neutral heavy leptons in $Z^{0}$ decays, Phys. Lett. B 295 (1992) 371 [INSPIRE].

[14] ATLAS collaboration, Search for heavy neutrinos and right-handed $W$ bosons in events with two leptons and jets in pp collisions at $\sqrt{s}=7 \mathrm{TeV}$ with the ATLAS detector, Eur. Phys. J. C 72 (2012) 2056 [arXiv:1203.5420] [InSPIRE].

[15] CMS collaboration, Search for heavy neutrinos and $W$ bosons with right-handed couplings in proton-proton collisions at $\sqrt{s}=8$ TeV, Eur. Phys. J. C 74 (2014) 3149 [arXiv:1407.3683] [INSPIRE].

[16] CMS collaboration, The CMS Experiment at the CERN LHC, 2008 JINST 3 S08004 [INSPIRE].

[17] T. Sjöstrand et al., An Introduction to PYTHIA 8.2, Comput. Phys. Commun. 191 (2015) 159 [arXiv: 1410.3012] [INSPIRE].

[18] M. Krämer, T. Plehn, M. Spira and P.M. Zerwas, Pair production of scalar leptoquarks at the CERN LHC, Phys. Rev. D 71 (2005) 057503 [hep-ph/0411038] [INSPIRE].

[19] M. Krämer, T. Plehn, M. Spira and P.M. Zerwas, Pair production of scalar leptoquarks at the Tevatron, Phys. Rev. Lett. 79 (1997) 341 [hep-ph/9704322] [INSPIRE].

[20] F. del Aguila, J.A. Aguilar-Saavedra and R. Pittau, Heavy neutrino signals at large hadron colliders, JHEP 10 (2007) 047 [hep-ph/0703261] [INSPIRE].

[21] F. Maltoni and T. Stelzer, MadEvent: Automatic event generation with MadGraph, JHEP 02 (2003) 027 [hep-ph/0208156] [INSPIRE].

[22] P. Nason, A New method for combining NLO QCD with shower Monte Carlo algorithms, JHEP 11 (2004) 040 [hep-ph/0409146] [INSPIRE].

[23] S. Frixione, P. Nason and C. Oleari, Matching NLO QCD computations with Parton Shower simulations: the POWHEG method, JHEP 11 (2007) 070 [arXiv:0709.2092] [INSPIRE].

[24] S. Alioli, P. Nason, C. Oleari and E. Re, A general framework for implementing NLO calculations in shower Monte Carlo programs: the POWHEG BOX, JHEP 06 (2010) 043 [arXiv: 1002.2581] [INSPIRE].

[25] NNPDF collaboration, R.D. Ball et al., Parton distributions for the LHC Run II, JHEP 04 (2015) 040 [arXiv: 1410.8849] [INSPIRE].

[26] CMS collaboration, Event generator tunes obtained from underlying event and multiparton scattering measurements, Eur. Phys. J. C 76 (2016) 155 [arXiv:1512.00815] [InSPIRE].

[27] GEANT4 collaboration, S. Agostinelli et al., GEANT4 - a simulation toolkit, Nucl. Instrum. Meth. A 506 (2003) 250 [inSPIRE]. 
[28] CMS collaboration, Particle-Flow Event Reconstruction in CMS and Performance for Jets, Taus and MET, CMS-PAS-PFT-09-001 (2009) [INSPIRE].

[29] CMS collaboration, Commissioning of the Particle-flow Event Reconstruction with the first LHC collisions recorded in the CMS detector, CMS-PAS-PFT-10-001 (2010) [INSPIRE].

[30] CMS collaboration, Performance of Electron Reconstruction and Selection with the CMS Detector in Proton-Proton Collisions at $\sqrt{s}=8 \mathrm{TeV}, 2015$ JINST $10 \mathrm{P} 06005$ [arXiv: 1502 .02701] [INSPIRE].

[31] H. Voss, A. Höcker, J. Stelzer and F. Tegenfeldt, TMVA: Toolkit for Multivariate Data Analysis with ROOT, PoS (ACAT) 040 [physics/0703039] [INSPIRE].

[32] CMS collaboration, Performance of CMS muon reconstruction in pp collision events at $\sqrt{s}=7 \mathrm{TeV}, 2012$ JINST 7 P10002 [arXiv:1206.4071] [INSPIRE].

[33] CMS collaboration, Reconstruction and identification of $\tau$ lepton decays to hadrons and $\nu_{\tau}$ at CMS, 2016 JINST 11 P01019 [arXiv:1510.07488] [INSPIRE].

[34] CMS collaboration, Performance of reconstruction and identification of tau leptons in their decays to hadrons and tau neutrino in LHC Run-2, CMS-PAS-TAU-16-002 (2016) [INSPIRE].

[35] G.P. Salam, Towards Jetography, Eur. Phys. J. C 67 (2010) 637 [arXiv:0906.1833] [INSPIRE].

[36] M. Cacciari, G.P. Salam and G. Soyez, The Anti-k $k_{t}$ jet clustering algorithm, JHEP 04 (2008) 063 [arXiv: 0802.1189] [INSPIRE].

[37] CMS collaboration, Pileup Removal Algorithms, CMS-PAS-JME-14-001 (2014) [INSPIRE].

[38] CMS collaboration, Jet energy scale and resolution in the CMS experiment in pp collisions at $8 \mathrm{TeV}, 2017$ JINST 12 P02014 [arXiv:1607.03663] [INSPIRE].

[39] CMS collaboration, Pileup Jet Identification, CMS-PAS-JME-13-005 (2013) [inSPIRE].

[40] CMS collaboration, Performance of missing energy reconstruction in $13 \mathrm{TeV}$ pp collision data using the CMS detector, CMS-PAS-JME-16-004 (2016) [INSPIRE].

[41] CMS collaboration, Identification of b quark jets at the CMS Experiment in the LHC Run 2, CMS-PAS-BTV-15-001 (2016) [INSPIRE].

[42] CMS collaboration, Measurement of the differential cross section for top quark pair production in pp collisions at $\sqrt{s}=8 \mathrm{TeV}$, Eur. Phys. J. C 75 (2015) 542 [arXiv: 1505.04480] [INSPIRE].

[43] CMS collaboration, Measurement of differential top-quark pair production cross sections in pp colisions at $\sqrt{s}=7 \mathrm{TeV}$, Eur. Phys. J. C 73 (2013) 2339 [arXiv:1211.2220] [InSPIRE].

[44] CMS collaboration, CMS luminosity measurement for the 2015 data-taking period, CMS-PAS-LUM-15-001 (2016) [INSPIRE].

[45] CMS collaboration, Measurement of the Inclusive $W$ and $Z$ Production Cross Sections in pp Collisions at $\sqrt{s}=7 \mathrm{TeV}$, JHEP 10 (2011) 132 [arXiv:1107.4789] [INSPIRE].

[46] CMS collaboration, Performance of $\tau$-lepton reconstruction and identification in CMS, 2012 JINST 7 P01001 [arXiv: 1109.6034] [INSPIRE].

[47] M. Czakon and A. Mitov, Top++: A Program for the Calculation of the Top-Pair Cross-Section at Hadron Colliders, Comput. Phys. Commun. 185 (2014) 2930 [arXiv: 1112.5675] [INSPIRE]. 
[48] M. Botje et al., The PDF4LHC Working Group Interim Recommendations, arXiv: 1101.0538 [INSPIRE].

[49] CMS collaboration, Measurement of $W^{+} W^{-}$and $Z Z$ production cross sections in $p p$ collisions at $\sqrt{s}=8 \mathrm{TeV}$, Phys. Lett. B 721 (2013) 190 [arXiv:1301.4698] [InSPIRE].

[50] S. Alekhin et al., The PDF 4LHC Working Group Interim Report, arXiv:1101.0536 [INSPIRE].

[51] P.M. Nadolsky et al., Implications of CTEQ global analysis for collider observables, Phys. Rev. D 78 (2008) 013004 [arXiv: 0802.0007] [InSPIRE].

[52] A.D. Martin, W.J. Stirling, R.S. Thorne and G. Watt, Update of parton distributions at NNLO, Phys. Lett. B 652 (2007) 292 [arXiv:0706.0459] [InSPIRE].

[53] M. Ubiali, NNPDF1.0 parton set for the LHC, in proceedings of the 14th High-Energy Physics International Conference on Quantum Chromodynamics (QCD 08), Montpellier, France, 7-12 July 2008, p. 62 [Nucl. Phys. Proc. Suppl. 186 (2009) 62] [arXiv:0809.3716] [INSPIRE].

[54] CMS collaboration, Determination of Jet Energy Calibration and Transverse Momentum Resolution in CMS, 2011 JINST 6 P11002 [arXiv:1107.4277] [INSPIRE].

[55] R.J. Barlow and C. Beeston, Fitting using finite Monte Carlo samples, Comput. Phys. Commun. 77 (1993) 219 [INSPIRE].

[56] T. Junk, Confidence level computation for combining searches with small statistics, Nucl. Instrum. Meth. A 434 (1999) 435 [hep-ex/9902006] [INSPIRE].

[57] A.L. Read, Presentation of search results: The CLs technique, J. Phys. G 28 (2002) 2693 [INSPIRE]. 


\section{The CMS collaboration}

\section{Yerevan Physics Institute, Yerevan, Armenia}

A.M. Sirunyan, A. Tumasyan

\section{Institut für Hochenergiephysik, Wien, Austria}

W. Adam, E. Asilar, T. Bergauer, J. Brandstetter, E. Brondolin, M. Dragicevic, J. Erö, M. Flechl, M. Friedl, R. Frühwirth ${ }^{1}$, V.M. Ghete, C. Hartl, N. Hörmann, J. Hrubec, M. Jeitler ${ }^{1}$, A. König, I. Krätschmer, D. Liko, T. Matsushita, I. Mikulec, D. Rabady, N. Rad, B. Rahbaran, H. Rohringer, J. Schieck ${ }^{1}$, J. Strauss, W. Waltenberger, C.-E. Wulz ${ }^{1}$

Institute for Nuclear Problems, Minsk, Belarus

O. Dvornikov, V. Makarenko, V. Mossolov, J. Suarez Gonzalez, V. Zykunov

National Centre for Particle and High Energy Physics, Minsk, Belarus

N. Shumeiko

\section{Universiteit Antwerpen, Antwerpen, Belgium}

S. Alderweireldt, E.A. De Wolf, X. Janssen, J. Lauwers, M. Van De Klundert, H. Van Haevermaet, P. Van Mechelen, N. Van Remortel, A. Van Spilbeeck

\section{Vrije Universiteit Brussel, Brussel, Belgium}

S. Abu Zeid, F. Blekman, J. D'Hondt, N. Daci, I. De Bruyn, K. Deroover, S. Lowette, S. Moortgat, L. Moreels, A. Olbrechts, Q. Python, K. Skovpen, S. Tavernier, W. Van Doninck, P. Van Mulders, I. Van Parijs

Université Libre de Bruxelles, Bruxelles, Belgium

H. Brun, B. Clerbaux, G. De Lentdecker, H. Delannoy, G. Fasanella, L. Favart, R. Goldouzian, A. Grebenyuk, G. Karapostoli, T. Lenzi, A. Léonard, J. Luetic, T. Maerschalk, A. Marinov, A. Randle-conde, T. Seva, C. Vander Velde, P. Vanlaer, D. Vannerom, R. Yonamine, F. Zenoni, F. Zhang ${ }^{2}$

\section{Ghent University, Ghent, Belgium}

T. Cornelis, D. Dobur, A. Fagot, M. Gul, I. Khvastunov, D. Poyraz, S. Salva, R. Schöfbeck, M. Tytgat, W. Van Driessche, E. Yazgan, N. Zaganidis

Université Catholique de Louvain, Louvain-la-Neuve, Belgium

H. Bakhshiansohi, C. Beluffi ${ }^{3}$, O. Bondu, S. Brochet, G. Bruno, A. Caudron, S. De Visscher, C. Delaere, M. Delcourt, B. Francois, A. Giammanco, A. Jafari, M. Komm, G. Krintiras, V. Lemaitre, A. Magitteri, A. Mertens, M. Musich, K. Piotrzkowski, L. Quertenmont, M. Selvaggi, M. Vidal Marono, S. Wertz

Université de Mons, Mons, Belgium

N. Beliy

Centro Brasileiro de Pesquisas Fisicas, Rio de Janeiro, Brazil

W.L. Aldá Júnior, F.L. Alves, G.A. Alves, L. Brito, C. Hensel, A. Moraes, M.E. Pol, P. Rebello Teles 
Universidade do Estado do Rio de Janeiro, Rio de Janeiro, Brazil

E. Belchior Batista Das Chagas, W. Carvalho, J. Chinellato ${ }^{4}$, A. Custódio, E.M. Da Costa, G.G. Da Silveira ${ }^{5}$, D. De Jesus Damiao, C. De Oliveira Martins, S. Fonseca De Souza, L.M. Huertas Guativa, H. Malbouisson, D. Matos Figueiredo, C. Mora Herrera, L. Mundim, H. Nogima, W.L. Prado Da Silva, A. Santoro, A. Sznajder, E.J. Tonelli Manganote ${ }^{4}$,

F. Torres Da Silva De Araujo, A. Vilela Pereira

Universidade Estadual Paulista ${ }^{a}$, Universidade Federal do ABC ${ }^{b}$, São Paulo, Brazil

S. Ahuja ${ }^{a}$, C.A. Bernardes ${ }^{a}$, S. Dogra ${ }^{a}$, T.R. Fernandez Perez Tomei $^{a}$, E.M. Gregores ${ }^{b}$, P.G. Mercadante ${ }^{b}$, C.S. Moon ${ }^{a}$, S.F. Novaes ${ }^{a}$, Sandra S. Padula ${ }^{a}$, D. Romero Abad ${ }^{b}$, J.C. Ruiz Vargas ${ }^{a}$

Institute for Nuclear Research and Nuclear Energy, Sofia, Bulgaria

A. Aleksandrov, R. Hadjiiska, P. Iaydjiev, M. Rodozov, S. Stoykova, G. Sultanov, M. Vutova

University of Sofia, Sofia, Bulgaria

A. Dimitrov, I. Glushkov, L. Litov, B. Pavlov, P. Petkov

Beihang University, Beijing, China

W. Fang ${ }^{6}$

Institute of High Energy Physics, Beijing, China

M. Ahmad, J.G. Bian, G.M. Chen, H.S. Chen, M. Chen, Y. Chen ${ }^{7}$, T. Cheng, C.H. Jiang,

D. Leggat, Z. Liu, F. Romeo, M. Ruan, S.M. Shaheen, A. Spiezia, J. Tao, C. Wang,

Z. Wang, H. Zhang, J. Zhao

State Key Laboratory of Nuclear Physics and Technology, Peking University, Beijing, China

Y. Ban, G. Chen, Q. Li, S. Liu, Y. Mao, S.J. Qian, D. Wang, Z. Xu

Universidad de Los Andes, Bogota, Colombia

C. Avila, A. Cabrera, L.F. Chaparro Sierra, C. Florez, J.P. Gomez, C.F. González Hernández, J.D. Ruiz Alvarez ${ }^{8}$, J.C. Sanabria

University of Split, Faculty of Electrical Engineering, Mechanical Engineering and Naval Architecture, Split, Croatia

N. Godinovic, D. Lelas, I. Puljak, P.M. Ribeiro Cipriano, T. Sculac

University of Split, Faculty of Science, Split, Croatia

Z. Antunovic, M. Kovac

Institute Rudjer Boskovic, Zagreb, Croatia

V. Brigljevic, D. Ferencek, K. Kadija, B. Mesic, T. Susa

University of Cyprus, Nicosia, Cyprus

M.W. Ather, A. Attikis, G. Mavromanolakis, J. Mousa, C. Nicolaou, F. Ptochos, P.A. Razis, H. Rykaczewski 
Charles University, Prague, Czech Republic

M. Finger ${ }^{9}$, M. Finger Jr. ${ }^{9}$

Universidad San Francisco de Quito, Quito, Ecuador

E. Carrera Jarrin

Academy of Scientific Research and Technology of the Arab Republic of Egypt, Egyptian Network of High Energy Physics, Cairo, Egypt

A. Ellithi Kamel ${ }^{10}$, M.A. Mahmoud ${ }^{11,12}$, A. Radi ${ }^{12,13}$

National Institute of Chemical Physics and Biophysics, Tallinn, Estonia

M. Kadastik, L. Perrini, M. Raidal, A. Tiko, C. Veelken

Department of Physics, University of Helsinki, Helsinki, Finland

P. Eerola, J. Pekkanen, M. Voutilainen

Helsinki Institute of Physics, Helsinki, Finland

J. Härkönen, T. Järvinen, V. Karimäki, R. Kinnunen, T. Lampén, K. Lassila-Perini,

S. Lehti, T. Lindén, P. Luukka, J. Tuominiemi, E. Tuovinen, L. Wendland

Lappeenranta University of Technology, Lappeenranta, Finland

J. Talvitie, T. Tuuva

IRFU, CEA, Université Paris-Saclay, Gif-sur-Yvette, France

M. Besancon, F. Couderc, M. Dejardin, D. Denegri, B. Fabbro, J.L. Faure, C. Favaro, F. Ferri, S. Ganjour, S. Ghosh, A. Givernaud, P. Gras, G. Hamel de Monchenault, P. Jarry, I. Kucher, E. Locci, M. Machet, J. Malcles, J. Rander, A. Rosowsky, M. Titov

Laboratoire Leprince-Ringuet, Ecole Polytechnique, IN2P3-CNRS, Palaiseau, France

A. Abdulsalam, I. Antropov, S. Baffioni, F. Beaudette, P. Busson, L. Cadamuro, E. Chapon, C. Charlot, O. Davignon, R. Granier de Cassagnac, M. Jo, S. Lisniak, P. Miné, M. Nguyen, C. Ochando, G. Ortona, P. Paganini, P. Pigard, S. Regnard, R. Salerno, Y. Sirois, A.G. Stahl Leiton, T. Strebler, Y. Yilmaz, A. Zabi, A. Zghiche

Institut Pluridisciplinaire Hubert Curien (IPHC), Université de Strasbourg, CNRS-IN2P3

J.-L. Agram ${ }^{14}$, J. Andrea, D. Bloch, J.-M. Brom, M. Buttignol, E.C. Chabert, N. Chanon, C. Collard, E. Conte ${ }^{14}$, X. Coubez, J.-C. Fontaine ${ }^{14}$, D. Gelé, U. Goerlach, A.-C. Le Bihan, P. Van Hove

Centre de Calcul de l'Institut National de Physique Nucleaire et de Physique des Particules, CNRS/IN2P3, Villeurbanne, France

S. Gadrat

Université de Lyon, Université Claude Bernard Lyon 1, CNRS-IN2P3, Institut de Physique Nucléaire de Lyon, Villeurbanne, France

S. Beauceron, C. Bernet, G. Boudoul, C.A. Carrillo Montoya, R. Chierici, D. Contardo, B. Courbon, P. Depasse, H. El Mamouni, J. Fay, S. Gascon, M. Gouzevitch, G. Grenier, 
B. Ille, F. Lagarde, I.B. Laktineh, M. Lethuillier, L. Mirabito, A.L. Pequegnot, S. Perries, A. Popov ${ }^{15}$, V. Sordini, M. Vander Donckt, P. Verdier, S. Viret

\section{Georgian Technical University, Tbilisi, Georgia}

A. Khvedelidze ${ }^{9}$

Tbilisi State University, Tbilisi, Georgia

Z. Tsamalaidze ${ }^{9}$

RWTH Aachen University, I. Physikalisches Institut, Aachen, Germany

C. Autermann, S. Beranek, L. Feld, M.K. Kiesel, K. Klein, M. Lipinski, M. Preuten, C. Schomakers, J. Schulz, T. Verlage

RWTH Aachen University, III. Physikalisches Institut A, Aachen, Germany A. Albert, M. Brodski, E. Dietz-Laursonn, D. Duchardt, M. Endres, M. Erdmann, S. Erdweg, T. Esch, R. Fischer, A. Güth, M. Hamer, T. Hebbeker, C. Heidemann, K. Hoepfner, S. Knutzen, M. Merschmeyer, A. Meyer, P. Millet, S. Mukherjee, M. Olschewski, K. Padeken, T. Pook, M. Radziej, H. Reithler, M. Rieger, F. Scheuch, L. Sonnenschein, D. Teyssier, S. Thüer

RWTH Aachen University, III. Physikalisches Institut B, Aachen, Germany

V. Cherepanov, G. Flügge, B. Kargoll, T. Kress, A. Künsken, J. Lingemann, T. Müller, A. Nehrkorn, A. Nowack, C. Pistone, O. Pooth, A. Stahl ${ }^{16}$

\section{Deutsches Elektronen-Synchrotron, Hamburg, Germany}

M. Aldaya Martin, T. Arndt, C. Asawatangtrakuldee, K. Beernaert, O. Behnke, U. Behrens, A.A. Bin Anuar, K. Borras ${ }^{17}$, A. Campbell, P. Connor, C. ContrerasCampana, F. Costanza, C. Diez Pardos, G. Dolinska, G. Eckerlin, D. Eckstein, T. Eichhorn, E. Eren, E. Gallo ${ }^{18}$, J. Garay Garcia, A. Geiser, A. Gizhko, J.M. Grados Luyando, A. Grohsjean, P. Gunnellini, A. Harb, J. Hauk, M. Hempel ${ }^{19}$, H. Jung, A. Kalogeropoulos, O. Karacheban ${ }^{19}$, M. Kasemann, J. Keaveney, C. Kleinwort, I. Korol, D. Krücker, W. Lange, A. Lelek, T. Lenz, J. Leonard, K. Lipka, A. Lobanov, W. Lohmann ${ }^{19}$, R. Mankel, I.-A. Melzer-Pellmann, A.B. Meyer, G. Mittag, J. Mnich, A. Mussgiller, D. Pitzl, R. Placakyte, A. Raspereza, B. Roland, M.Ö. Sahin, P. Saxena, T. SchoernerSadenius, S. Spannagel, N. Stefaniuk, G.P. Van Onsem, R. Walsh, C. Wissing

\section{University of Hamburg, Hamburg, Germany}

V. Blobel, M. Centis Vignali, A.R. Draeger, T. Dreyer, E. Garutti, D. Gonzalez, J. Haller, M. Hoffmann, A. Junkes, R. Klanner, R. Kogler, N. Kovalchuk, T. Lapsien, I. Marchesini, D. Marconi, M. Meyer, M. Niedziela, D. Nowatschin, F. Pantaleo ${ }^{16}$, T. Peiffer, A. Perieanu, C. Scharf, P. Schleper, A. Schmidt, S. Schumann, J. Schwandt, H. Stadie, G. Steinbrück, F.M. Stober, M. Stöver, H. Tholen, D. Troendle, E. Usai, L. Vanelderen, A. Vanhoefer, B. Vormwald

\section{Institut für Experimentelle Kernphysik, Karlsruhe, Germany}

M. Akbiyik, C. Barth, S. Baur, C. Baus, J. Berger, E. Butz, R. Caspart, T. Chwalek, F. Colombo, W. De Boer, A. Dierlamm, S. Fink, B. Freund, R. Friese, M. Giffels, A. Gilbert, 
P. Goldenzweig, D. Haitz, F. Hartmann ${ }^{16}$, S.M. Heindl, U. Husemann, F. Kassel ${ }^{16}$, I. Katkov ${ }^{15}$, S. Kudella, H. Mildner, M.U. Mozer, Th. Müller, M. Plagge, G. Quast, K. Rabbertz, S. Röcker, F. Roscher, M. Schröder, I. Shvetsov, G. Sieber, H.J. Simonis,

R. Ulrich, S. Wayand, M. Weber, T. Weiler, S. Williamson, C. Wöhrmann, R. Wolf

Institute of Nuclear and Particle Physics (INPP), NCSR Demokritos, Aghia Paraskevi, Greece

G. Anagnostou, G. Daskalakis, T. Geralis, V.A. Giakoumopoulou, A. Kyriakis, D. Loukas, I. Topsis-Giotis

National and Kapodistrian University of Athens, Athens, Greece

S. Kesisoglou, A. Panagiotou, N. Saoulidou, E. Tziaferi

University of Ioánnina, Ioánnina, Greece

I. Evangelou, G. Flouris, C. Foudas, P. Kokkas, N. Loukas, N. Manthos, I. Papadopoulos, E. Paradas

MTA-ELTE Lendület CMS Particle and Nuclear Physics Group, Eötvös Loránd University, Budapest, Hungary

N. Filipovic, G. Pasztor

Wigner Research Centre for Physics, Budapest, Hungary

G. Bencze, C. Hajdu, D. Horvath ${ }^{20}$, F. Sikler, V. Veszpremi, G. Vesztergombi ${ }^{21}$, A.J. Zsigmond

Institute of Nuclear Research ATOMKI, Debrecen, Hungary

N. Beni, S. Czellar, J. Karancsi22, A. Makovec, J. Molnar, Z. Szillasi

Institute of Physics, University of Debrecen

M. Bartók ${ }^{21}$, P. Raics, Z.L. Trocsanyi, B. Ujvari

Indian Institute of Science (IISc)

J.R. Komaragiri

National Institute of Science Education and Research, Bhubaneswar, India

S. Bahinipati ${ }^{23}$, S. Bhowmik ${ }^{24}$, S. Choudhury ${ }^{25}$, P. Mal, K. Mandal, A. Nayak ${ }^{26}$, D.K. Sahoo ${ }^{23}$, N. Sahoo, S.K. Swain

Panjab University, Chandigarh, India

S. Bansal, S.B. Beri, V. Bhatnagar, R. Chawla, U.Bhawandeep, A.K. Kalsi, A. Kaur, M. Kaur, R. Kumar, P. Kumari, A. Mehta, M. Mittal, J.B. Singh, G. Walia

University of Delhi, Delhi, India

Ashok Kumar, A. Bhardwaj, B.C. Choudhary, R.B. Garg, S. Keshri, S. Malhotra, M. Naimuddin, K. Ranjan, R. Sharma, V. Sharma

Saha Institute of Nuclear Physics, Kolkata, India

R. Bhattacharya, S. Bhattacharya, K. Chatterjee, S. Dey, S. Dutt, S. Dutta, S. Ghosh,

N. Majumdar, A. Modak, K. Mondal, S. Mukhopadhyay, S. Nandan, A. Purohit, A. Roy,

D. Roy, S. Roy Chowdhury, S. Sarkar, M. Sharan, S. Thakur 
Indian Institute of Technology Madras, Madras, India

P.K. Behera

Bhabha Atomic Research Centre, Mumbai, India

R. Chudasama, D. Dutta, V. Jha, V. Kumar, A.K. Mohanty ${ }^{16}$, P.K. Netrakanti, L.M. Pant, P. Shukla, A. Topkar

Tata Institute of Fundamental Research-A, Mumbai, India

T. Aziz, S. Dugad, G. Kole, B. Mahakud, S. Mitra, G.B. Mohanty, B. Parida, N. Sur, B. Sutar

Tata Institute of Fundamental Research-B, Mumbai, India

S. Banerjee, R.K. Dewanjee, S. Ganguly, M. Guchait, Sa. Jain, S. Kumar, M. Maity ${ }^{24}$, G. Majumder, K. Mazumdar, T. Sarkar ${ }^{24}$, N. Wickramage ${ }^{27}$

Indian Institute of Science Education and Research (IISER), Pune, India

S. Chauhan, S. Dube, V. Hegde, A. Kapoor, K. Kothekar, S. Pandey, A. Rane, S. Sharma

Institute for Research in Fundamental Sciences (IPM), Tehran, Iran

S. Chenarani ${ }^{28}$, E. Eskandari Tadavani, S.M. Etesami ${ }^{28}$, M. Khakzad, M. Mohammadi Najafabadi, M. Naseri, S. Paktinat Mehdiabadi ${ }^{29}$, F. Rezaei Hosseinabadi, B. Safarzadeh ${ }^{30}$,

M. Zeinali

University College Dublin, Dublin, Ireland

M. Felcini, M. Grunewald

INFN Sezione di Bari ${ }^{a}$, Università di Bari ${ }^{b}$, Politecnico di Bari ${ }^{c}$, Bari, Italy M. Abbrescia ${ }^{a, b}$, C. Calabria ${ }^{a, b}$, C. Caputo $^{a, b}$, A. Colaleo ${ }^{a}$, D. Creanza ${ }^{a, c}$, L. Cristella $^{a, b}$, N. De Filippis ${ }^{a, c}$, M. De Palma ${ }^{a, b}$, L. Fiore ${ }^{a}$, G. Iaselli ${ }^{a, c}$, G. Maggi ${ }^{a, c}$, M. Maggi $^{a}$, G. Miniello ${ }^{a, b}$, S. My ${ }^{a, b}$, S. Nuzzo ${ }^{a, b}$, A. Pompili ${ }^{a, b}$, G. Pugliese ${ }^{a, c}$, R. Radogna ${ }^{a, b}$, A. Ranieri ${ }^{a}$, G. Selvaggi ${ }^{a, b}$, A. Sharma ${ }^{a}$, L. Silvestris ${ }^{a, 16}$, R. Venditti ${ }^{a, b}$, P. Verwilligen $^{a}$

INFN Sezione di Bologna ${ }^{a}$, Università di Bologna ${ }^{b}$, Bologna, Italy

G. Abbiendi ${ }^{a}$, C. Battilana, D. Bonacorsi ${ }^{a, b}$, S. Braibant-Giacomelli ${ }^{a, b}$, L. Brigliadori $^{a, b}$, R. Campanini ${ }^{a, b}$, P. Capiluppi ${ }^{a, b}$, A. Castro ${ }^{a, b}$, F.R. Cavallo ${ }^{a}$, S.S. Chhibra ${ }^{a, b}$, G. Codispoti ${ }^{a, b}$, M. Cuffiani ${ }^{a, b}$, G.M. Dallavalle ${ }^{a}$, F. Fabbri $^{a}$, A. Fanfani ${ }^{a, b}$, D. Fasanella ${ }^{a, b}$, P. Giacomelli ${ }^{a}$, C. Grandi ${ }^{a}$, L. Guiducci ${ }^{a}, b$, S. Marcellini ${ }^{a}$, G. Masetti ${ }^{a}$, A. Montanari ${ }^{a}$,

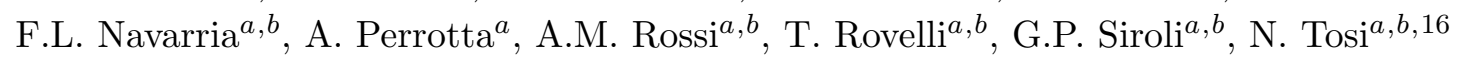

INFN Sezione di Catania ${ }^{a}$, Università di Catania ${ }^{b}$, Catania, Italy

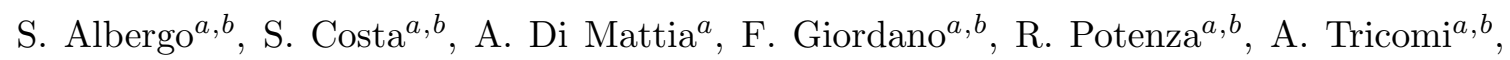
C. Tuve ${ }^{a, b}$

INFN Sezione di Firenze ${ }^{a}$, Università di Firenze ${ }^{b}$, Firenze, Italy

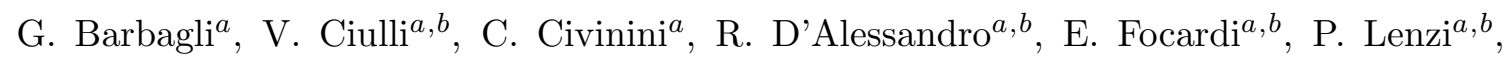

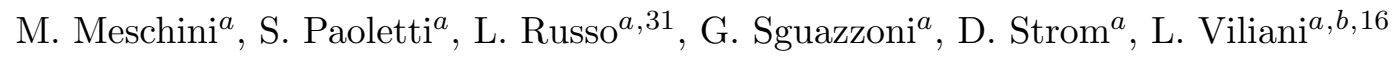

INFN Laboratori Nazionali di Frascati, Frascati, Italy

L. Benussi, S. Bianco, F. Fabbri, D. Piccolo, F. Primavera ${ }^{16}$ 
INFN Sezione di Genova ${ }^{a}$, Università di Genova ${ }^{b}$, Genova, Italy

V. Calvelli ${ }^{a, b}$, F. Ferro ${ }^{a}$, M.R. Monge ${ }^{a, b}$, E. Robutti ${ }^{a}$, S. Tosi ${ }^{a, b}$

INFN Sezione di Milano-Bicocca ${ }^{a}$, Università di Milano-Bicocca ${ }^{b}$, Milano, Italy

L. Brianza ${ }^{a, b, 16}$, F. Brivio ${ }^{a, b}$, V. Ciriolo, M.E. Dinardo ${ }^{a, b}$, S. Fiorendi ${ }^{a, b}, 16$, S. Gennai $^{a}$,

A. Ghezzi ${ }^{a, b}$, P. Govoni ${ }^{a, b}$, M. Malberti ${ }^{a}, b$, S. Malvezzi ${ }^{a}$, R.A. Manzoni ${ }^{a, b}$, D. Menasce $^{a}$, L. Moroni ${ }^{a}$, M. Paganoni ${ }^{a, b}$, D. Pedrini ${ }^{a}$, S. Pigazzini ${ }^{a, b}$, S. Ragazzi ${ }^{a, b}$, T. Tabarelli de Fatis $^{a, b}$

INFN Sezione di Napoli ${ }^{a}$, Università di Napoli 'Federico II' ${ }^{b}$, Napoli, Italy, Università della Basilicata ${ }^{c}$, Potenza, Italy, Università G. Marconi ${ }^{d}$, Roma, Italy

S. Buontempo ${ }^{a}$, N. Cavallo ${ }^{a, c}$, G. De Nardo, S. Di Guida ${ }^{a, d, 16}$, M. Esposito $^{a, b}$, F. Fabozzi ${ }^{a, c}$, F. Fienga ${ }^{a, b}$, A.O.M. Iorio ${ }^{a, b}$, G. Lanza ${ }^{a}$, L. Lista ${ }^{a}$, S. Meola ${ }^{a, d}{ }^{16}$, P. Paolucci ${ }^{a, 16}$, C. Sciacca ${ }^{a, b}$, F. Thyssen ${ }^{a}$

INFN Sezione di Padova ${ }^{a}$, Università di Padova ${ }^{b}$, Padova, Italy, Università di Trento ${ }^{c}$, Trento, Italy

P. Azzi ${ }^{a, 16}$, N. Bacchetta ${ }^{a}$, L. Benato ${ }^{a, b}$, A. Boletti $^{a, b}$, A. Carvalho Antunes De Oliveira ${ }^{a, b}$, P. Checchia ${ }^{a}$, M. Dall'Osso ${ }^{a, b}$, P. De Castro Manzano ${ }^{a}$, T. Dorigo ${ }^{a}$, U. Dosselli ${ }^{a}$, S. Fantinel ${ }^{a}$, F. Fanzago ${ }^{a}$, F. Gasparini ${ }^{a, b}$, U. Gasparini ${ }^{a, b}$, A. Gozzelino ${ }^{a}$, S. Lacaprara ${ }^{a}$, M. Margoni ${ }^{a, b}$, A.T. Meneguzzo ${ }^{a, b}$, J. Pazzini ${ }^{a, b}$, N. Pozzobon ${ }^{a, b}$, P. Ronchese ${ }^{a, b}$, F. Simonetto ${ }^{a, b}$, E. Torassa $^{a}$, S. Ventura ${ }^{a}$, M. Zanetti ${ }^{a, b}$, P. Zotto $^{a, b}$

INFN Sezione di Pavia ${ }^{a}$, Università di Pavia ${ }^{b}$, Pavia, Italy

A. Braghieri ${ }^{a}$, F. Fallavollita ${ }^{a, b}$, A. Magnani ${ }^{a, b}$, P. Montagna ${ }^{a, b}$, S.P. Ratti ${ }^{a, b}$, V. Re $^{a}$, C. Riccardi ${ }^{a, b}$, P. Salvini ${ }^{a}$, I. Vai ${ }^{a}, b$, P. Vitulo ${ }^{a, b}$

INFN Sezione di Perugia ${ }^{a}$, Università di Perugia ${ }^{b}$, Perugia, Italy

L. Alunni Solestizi ${ }^{a, b}$, G.M. Bilei ${ }^{a}$, D. Ciangottini ${ }^{a, b}$, L. Fanò ${ }^{a}, b$ P. Lariccia $^{a, b}$,

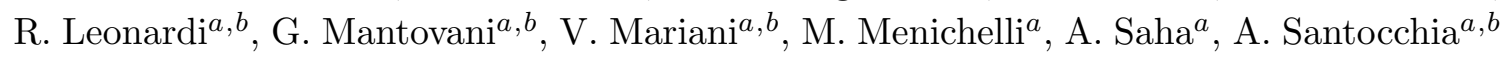

INFN Sezione di Pisa ${ }^{a}$, Università di Pisa ${ }^{b}$, Scuola Normale Superiore di Pisa ${ }^{c}$, Pisa, Italy

K. Androsov ${ }^{a}, 31$, P. Azzurri ${ }^{a}, 16$, G. Bagliesi ${ }^{a}$, J. Bernardini ${ }^{a}$, T. Boccali ${ }^{a}$, R. Castaldi ${ }^{a}$, M.A. Ciocci ${ }^{a}, 31$, R. Dell'Orso ${ }^{a}$, S. Donato ${ }^{a, c}$, G. Fedi, A. Giassi ${ }^{a}$, M.T. Grippo $^{a, 31}$,

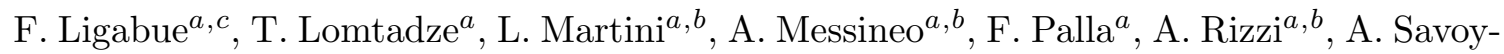

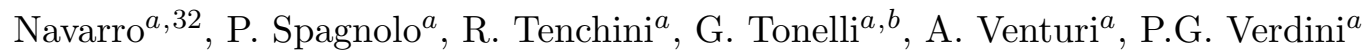

INFN Sezione di Roma ${ }^{a}$, Università di Roma ${ }^{b}$, Roma, Italy

L. Barone ${ }^{a, b}$, F. Cavallari ${ }^{a}$, M. Cipriani ${ }^{a, b}$, D. Del Re ${ }^{a, b, 16}$, M. Diemoz $^{a}$, S. Gelli ${ }^{a, b}$, E. Longo $^{a, b}$, F. Margaroli ${ }^{a, b}$, B. Marzocchi ${ }^{a, b}$, P. Meridiani ${ }^{a}$, G. Organtini ${ }^{a, b}$,

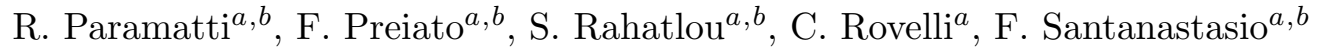


INFN Sezione di Torino ${ }^{a}$, Università di Torino ${ }^{b}$, Torino, Italy, Università del Piemonte Orientale ${ }^{c}$, Novara, Italy

N. Amapane ${ }^{a, b}$, R. Arcidiacono ${ }^{a, c, 16}$, S. Argiro ${ }^{a, b}$, M. Arneodo ${ }^{a, c}$, N. Bartosik ${ }^{a}$, R. Bellan ${ }^{a, b}$, C. Biino ${ }^{a}$, N. Cartiglia ${ }^{a}$, F. Cenna ${ }^{a, b}$, M. Costa ${ }^{a, b}$, R. Covarellia ${ }^{a, b}$, A. Degano ${ }^{a, b}$, N. Demaria ${ }^{a}$, L. Finco ${ }^{a, b}$, B. Kiani ${ }^{a, b}$, C. Mariotti ${ }^{a}$, S. Maselli ${ }^{a}$,

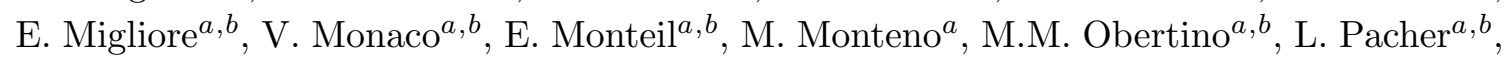
N. Pastrone ${ }^{a}$, M. Pelliccioni ${ }^{a}$, G.L. Pinna Angioni ${ }^{a, b}$, F. Ravera ${ }^{a, b}$, A. Romero ${ }^{a, b}$, M. Ruspa ${ }^{a, c}$, R. Sacchi ${ }^{a, b}$, K. Shchelina ${ }^{a, b}$, V. Sola ${ }^{a}$, A. Solano ${ }^{a, b}$, A. Staiano ${ }^{a}$, P. Traczyk ${ }^{a, b}$

INFN Sezione di Trieste ${ }^{a}$, Università di Trieste ${ }^{b}$, Trieste, Italy

S. Belforte ${ }^{a}$, M. Casarsa ${ }^{a}$, F. Cossutti ${ }^{a}$, G. Della Ricca ${ }^{a}, b$, A. Zanetti ${ }^{a}$

Kyungpook National University, Daegu, Korea

D.H. Kim, G.N. Kim, M.S. Kim, S. Lee, S.W. Lee, Y.D. Oh, S. Sekmen, D.C. Son, Y.C. Yang

Chonbuk National University, Jeonju, Korea

A. Lee

Chonnam National University, Institute for Universe and Elementary Particles, Kwangju, Korea

H. Kim

Hanyang University, Seoul, Korea

J.A. Brochero Cifuentes, T.J. Kim

Korea University, Seoul, Korea

S. Cho, S. Choi, Y. Go, D. Gyun, S. Ha, B. Hong, Y. Jo, Y. Kim, K. Lee, K.S. Lee, S. Lee, J. Lim, S.K. Park, Y. Roh

Seoul National University, Seoul, Korea

J. Almond, J. Kim, H. Lee, S.B. Oh, B.C. Radburn-Smith, S.h. Seo, U.K. Yang, H.D. Yoo, G.B. Yu

University of Seoul, Seoul, Korea

M. Choi, H. Kim, J.H. Kim, J.S.H. Lee, I.C. Park, G. Ryu, M.S. Ryu

Sungkyunkwan University, Suwon, Korea

Y. Choi, J. Goh, C. Hwang, J. Lee, I. Yu

Vilnius University, Vilnius, Lithuania

V. Dudenas, A. Juodagalvis, J. Vaitkus

National Centre for Particle Physics, Universiti Malaya, Kuala Lumpur, Malaysia

I. Ahmed, Z.A. Ibrahim, M.A.B. Md Ali ${ }^{33}$, F. Mohamad Idris ${ }^{34}$, W.A.T. Wan Abdullah, M.N. Yusli, Z. Zolkapli 
Centro de Investigacion y de Estudios Avanzados del IPN, Mexico City, Mexico H. Castilla-Valdez, E. De La Cruz-Burelo, I. Heredia-De La Cruz ${ }^{35}$, A. Hernandez-Almada, R. Lopez-Fernandez, R. Magaña Villalba, J. Mejia Guisao, A. Sanchez-Hernandez

Universidad Iberoamericana, Mexico City, Mexico

S. Carrillo Moreno, C. Oropeza Barrera, F. Vazquez Valencia

Benemerita Universidad Autonoma de Puebla, Puebla, Mexico

S. Carpinteyro, I. Pedraza, H.A. Salazar Ibarguen, C. Uribe Estrada

Universidad Autónoma de San Luis Potosí, San Luis Potosí, Mexico

A. Morelos Pineda

University of Auckland, Auckland, New Zealand

D. Krofcheck

University of Canterbury, Christchurch, New Zealand

P.H. Butler

National Centre for Physics, Quaid-I-Azam University, Islamabad, Pakistan

A. Ahmad, M. Ahmad, Q. Hassan, H.R. Hoorani, W.A. Khan, A. Saddique, M.A. Shah, M. Shoaib, M. Waqas

National Centre for Nuclear Research, Swierk, Poland

H. Bialkowska, M. Bluj, B. Boimska, T. Frueboes, M. Górski, M. Kazana, K. Nawrocki,

K. Romanowska-Rybinska, M. Szleper, P. Zalewski

Institute of Experimental Physics, Faculty of Physics, University of Warsaw, Warsaw, Poland

K. Bunkowski, A. Byszuk ${ }^{36}$, K. Doroba, A. Kalinowski, M. Konecki, J. Krolikowski, M. Misiura, M. Olszewski, M. Walczak

Laboratório de Instrumentação e Física Experimental de Partículas, Lisboa, Portugal

P. Bargassa, C. Beirão Da Cruz E Silva, B. Calpas, A. Di Francesco, P. Faccioli, M. Gallinaro, J. Hollar, N. Leonardo, L. Lloret Iglesias, M.V. Nemallapudi, J. Seixas, O. Toldaiev, D. Vadruccio, J. Varela

Joint Institute for Nuclear Research, Dubna, Russia

S. Afanasiev, P. Bunin, M. Gavrilenko, I. Golutvin, I. Gorbunov, A. Kamenev, V. Karjavin, A. Lanev, A. Malakhov, V. Matveev ${ }^{37,38}$, V. Palichik, V. Perelygin, S. Shmatov, S. Shulha, N. Skatchkov, V. Smirnov, N. Voytishin, A. Zarubin

Petersburg Nuclear Physics Institute, Gatchina (St. Petersburg), Russia

L. Chtchipounov, V. Golovtsov, Y. Ivanov, V. Kim ${ }^{39}$, E. Kuznetsova ${ }^{40}$, V. Murzin, V. Oreshkin, V. Sulimov, A. Vorobyev

Institute for Nuclear Research, Moscow, Russia

Yu. Andreev, A. Dermenev, S. Gninenko, N. Golubev, A. Karneyeu, M. Kirsanov, N. Krasnikov, A. Pashenkov, D. Tlisov, A. Toropin 
Institute for Theoretical and Experimental Physics, Moscow, Russia

V. Epshteyn, V. Gavrilov, N. Lychkovskaya, V. Popov, I. Pozdnyakov, G. Safronov, A. Spiridonov, M. Toms, E. Vlasov, A. Zhokin

Moscow Institute of Physics and Technology, Moscow, Russia

T. Aushev, A. Bylinkin ${ }^{38}$

National Research Nuclear University 'Moscow Engineering Physics Institute' (MEPhI), Moscow, Russia

M. Chadeeva ${ }^{41}$, O. Markin, V. Rusinov

P.N. Lebedev Physical Institute, Moscow, Russia

V. Andreev, M. Azarkin ${ }^{38}$, I. Dremin ${ }^{38}$, M. Kirakosyan, A. Leonidov ${ }^{38}$, A. Terkulov

Skobeltsyn Institute of Nuclear Physics, Lomonosov Moscow State University, Moscow, Russia

A. Baskakov, A. Belyaev, E. Boos, V. Bunichev, M. Dubinin ${ }^{42}$, L. Dudko, A. Ershov, V. Klyukhin, O. Kodolova, I. Lokhtin, I. Miagkov, S. Obraztsov, M. Perfilov, S. Petrushanko, V. Savrin

Novosibirsk State University (NSU), Novosibirsk, Russia

V. Blinov ${ }^{43}$, Y.Skovpen ${ }^{43}$, D. Shtol ${ }^{43}$

State Research Center of Russian Federation, Institute for High Energy Physics, Protvino, Russia

I. Azhgirey, I. Bayshev, S. Bitioukov, D. Elumakhov, V. Kachanov, A. Kalinin, D. Konstantinov, V. Krychkine, V. Petrov, R. Ryutin, A. Sobol, S. Troshin, N. Tyurin, A. Uzunian, A. Volkov

University of Belgrade, Faculty of Physics and Vinca Institute of Nuclear Sciences, Belgrade, Serbia

P. Adzic ${ }^{44}$, P. Cirkovic, D. Devetak, M. Dordevic, J. Milosevic, V. Rekovic

Centro de Investigaciones Energéticas Medioambientales y Tecnológicas (CIEMAT), Madrid, Spain

J. Alcaraz Maestre, M. Barrio Luna, E. Calvo, M. Cerrada, M. Chamizo Llatas, N. Colino, B. De La Cruz, A. Delgado Peris, A. Escalante Del Valle, C. Fernandez Bedoya, J.P. Fernández Ramos, J. Flix, M.C. Fouz, P. Garcia-Abia, O. Gonzalez Lopez, S. Goy Lopez, J.M. Hernandez, M.I. Josa, E. Navarro De Martino, A. Pérez-Calero Yzquierdo, J. Puerta Pelayo, A. Quintario Olmeda, I. Redondo, L. Romero, M.S. Soares

Universidad Autónoma de Madrid, Madrid, Spain

J.F. de Trocóniz, M. Missiroli, D. Moran

Universidad de Oviedo, Oviedo, Spain

J. Cuevas, J. Fernandez Menendez, I. Gonzalez Caballero, J.R. González Fernández, E. Palencia Cortezon, S. Sanchez Cruz, I. Suárez Andrés, P. Vischia, J.M. Vizan Garcia 
Instituto de Física de Cantabria (IFCA), CSIC-Universidad de Cantabria, Santander, Spain

I.J. Cabrillo, A. Calderon, E. Curras, M. Fernandez, J. Garcia-Ferrero, G. Gomez, A. Lopez Virto, J. Marco, C. Martinez Rivero, F. Matorras, J. Piedra Gomez, T. Rodrigo, A. RuizJimeno, L. Scodellaro, N. Trevisani, I. Vila, R. Vilar Cortabitarte

\section{CERN, European Organization for Nuclear Research, Geneva, Switzerland}

D. Abbaneo, E. Auffray, G. Auzinger, P. Baillon, A.H. Ball, D. Barney, P. Bloch, A. Bocci,

C. Botta, T. Camporesi, R. Castello, M. Cepeda, G. Cerminara, Y. Chen, A. Cimmino,

D. d'Enterria, A. Dabrowski, V. Daponte, A. David, M. De Gruttola, A. De Roeck, E. Di Marco ${ }^{45}$, M. Dobson, B. Dorney, T. du Pree, D. Duggan, M. Dünser, N. Dupont, A. Elliott-Peisert, P. Everaerts, S. Fartoukh, G. Franzoni, J. Fulcher, W. Funk, D. Gigi, K. Gill, M. Girone, F. Glege, D. Gulhan, S. Gundacker, M. Guthoff, P. Harris, J. Hegeman, V. Innocente, P. Janot, J. Kieseler, H. Kirschenmann, V. Knünz, A. Kornmayer ${ }^{16}$, M.J. Kortelainen, K. Kousouris, M. Krammer ${ }^{1}$, C. Lange, P. Lecoq, C. Lourenço, M.T. Lucchini, L. Malgeri, M. Mannelli, A. Martelli, F. Meijers, J.A. Merlin, S. Mersi, E. Meschi, P. Milenovic ${ }^{46}$, F. Moortgat, S. Morovic, M. Mulders, H. Neugebauer, S. Orfanelli, L. Orsini, L. Pape, E. Perez, M. Peruzzi, A. Petrilli, G. Petrucciani, A. Pfeiffer, M. Pierini, A. Racz, T. Reis, G. Rolandi ${ }^{47}$, M. Rovere, H. Sakulin, J.B. Sauvan, C. Schäfer, C. Schwick, M. Seidel, A. Sharma, P. Silva, P. Sphicas ${ }^{48}$, J. Steggemann, M. Stoye, Y. Takahashi, M. Tosi, D. Treille, A. Triossi, A. Tsirou, V. Veckalns ${ }^{49}$, G.I. Veres ${ }^{21}$, M. Verweij, N. Wardle, H.K. Wöhri, A. Zagozdzinska ${ }^{36}$, W.D. Zeuner

\section{Paul Scherrer Institut, Villigen, Switzerland}

W. Bertl, K. Deiters, W. Erdmann, R. Horisberger, Q. Ingram, H.C. Kaestli, D. Kotlinski, U. Langenegger, T. Rohe, S.A. Wiederkehr

\section{Institute for Particle Physics, ETH Zurich, Zurich, Switzerland}

F. Bachmair, L. Bäni, L. Bianchini, B. Casal, G. Dissertori, M. Dittmar, M. Donegà, C. Grab, C. Heidegger, D. Hits, J. Hoss, G. Kasieczka, W. Lustermann, B. Mangano, M. Marionneau, P. Martinez Ruiz del Arbol, M. Masciovecchio, M.T. Meinhard, D. Meister, F. Micheli, P. Musella, F. Nessi-Tedaldi, F. Pandolfi, J. Pata, F. Pauss, G. Perrin, L. Perrozzi, M. Quittnat, M. Rossini, M. Schönenberger, A. Starodumov ${ }^{50}$, V.R. Tavolaro, K. Theofilatos, R. Wallny

\section{Universität Zürich, Zurich, Switzerland}

T.K. Aarrestad, C. Amsler ${ }^{51}$, L. Caminada, M.F. Canelli, A. De Cosa, C. Galloni, A. Hinzmann, T. Hreus, B. Kilminster, J. Ngadiuba, D. Pinna, G. Rauco, P. Robmann, D. Salerno, C. Seitz, Y. Yang, A. Zucchetta

\section{National Central University, Chung-Li, Taiwan}

V. Candelise, T.H. Doan, Sh. Jain, R. Khurana, M. Konyushikhin, C.M. Kuo, W. Lin, A. Pozdnyakov, S.S. Yu 
National Taiwan University (NTU), Taipei, Taiwan

Arun Kumar, P. Chang, Y.H. Chang, Y. Chao, K.F. Chen, P.H. Chen, F. Fiori, W.-S. Hou, Y. Hsiung, Y.F. Liu, R.-S. Lu, M. Miñano Moya, E. Paganis, A. Psallidas, J.f. Tsai

Chulalongkorn University, Faculty of Science, Department of Physics, Bangkok, Thailand

B. Asavapibhop, G. Singh, N. Srimanobhas, N. Suwonjandee

Cukurova University - Physics Department, Science and Art Faculty

A. Adiguzel, M.N. Bakirci ${ }^{52}$, S. Damarseckin, Z.S. Demiroglu, C. Dozen, E. Eskut, S. Girgis, G. Gokbulut, Y. Guler, I. Hos ${ }^{53}$, E.E. Kangal ${ }^{54}$, O. Kara, U. Kiminsu, M. Oglakci, G. Onengut ${ }^{55}$, K. Ozdemir ${ }^{56}$, S. Ozturk ${ }^{52}$, A. Polatoz, D. Sunar Cerci ${ }^{57}$, S. Turkcapar, I.S. Zorbakir, C. Zorbilmez

Middle East Technical University, Physics Department, Ankara, Turkey

B. Bilin, S. Bilmis, B. Isildak ${ }^{58}$, G. Karapinar ${ }^{59}$, M. Yalvac, M. Zeyrek

Bogazici University, Istanbul, Turkey

E. Gülmez, M. Kaya ${ }^{60}$, O. Kaya ${ }^{61}$, E.A. Yetkin ${ }^{62}$, T. Yetkin ${ }^{63}$

Istanbul Technical University, Istanbul, Turkey

A. Cakir, K. Cankocak, S. Sen ${ }^{64}$

Institute for Scintillation Materials of National Academy of Science of Ukraine, Kharkov, Ukraine

B. Grynyov

National Scientific Center, Kharkov Institute of Physics and Technology, Kharkov, Ukraine

L. Levchuk, P. Sorokin

University of Bristol, Bristol, United Kingdom

R. Aggleton, F. Ball, L. Beck, J.J. Brooke, D. Burns, E. Clement, D. Cussans, H. Flacher, J. Goldstein, M. Grimes, G.P. Heath, H.F. Heath, J. Jacob, L. Kreczko, C. Lucas, D.M. Newbold ${ }^{65}$, S. Paramesvaran, A. Poll, T. Sakuma, S. Seif El Nasr-storey, D. Smith, V.J. Smith

Rutherford Appleton Laboratory, Didcot, United Kingdom

K.W. Bell, A. Belyaev ${ }^{66}$, C. Brew, R.M. Brown, L. Calligaris, D. Cieri, D.J.A. Cockerill, J.A. Coughlan, K. Harder, S. Harper, E. Olaiya, D. Petyt, C.H. Shepherd-Themistocleous, A. Thea, I.R. Tomalin, T. Williams

\section{Imperial College, London, United Kingdom}

M. Baber, R. Bainbridge, O. Buchmuller, A. Bundock, D. Burton, S. Casasso, M. Citron, D. Colling, L. Corpe, P. Dauncey, G. Davies, A. De Wit, M. Della Negra, R. Di Maria, P. Dunne, A. Elwood, D. Futyan, Y. Haddad, G. Hall, G. Iles, T. James, R. Lane, C. Laner, R. Lucas $^{65}$, L. Lyons, A.-M. Magnan, S. Malik, L. Mastrolorenzo, J. Nash, A. Nikitenko ${ }^{50}$, J. Pela, B. Penning, M. Pesaresi, D.M. Raymond, A. Richards, A. Rose, E. Scott, C. Seez, S. Summers, A. Tapper, K. Uchida, M. Vazquez Acosta ${ }^{67}$, T. Virdee ${ }^{16}$, J. Wright, S.C. Zenz 
Brunel University, Uxbridge, United Kingdom

J.E. Cole, P.R. Hobson, A. Khan, P. Kyberd, I.D. Reid, P. Symonds, L. Teodorescu, M. Turner

Baylor University, Waco, U.S.A.

A. Borzou, K. Call, J. Dittmann, K. Hatakeyama, H. Liu, N. Pastika

Catholic University of America

R. Bartek, A. Dominguez

The University of Alabama, Tuscaloosa, U.S.A.

A. Buccilli, S.I. Cooper, C. Henderson, P. Rumerio, C. West

Boston University, Boston, U.S.A.

D. Arcaro, A. Avetisyan, T. Bose, D. Gastler, D. Rankin, C. Richardson, J. Rohlf, L. Sulak,

D. Zou

Brown University, Providence, U.S.A.

G. Benelli, D. Cutts, A. Garabedian, J. Hakala, U. Heintz, J.M. Hogan, O. Jesus, K.H.M. Kwok, E. Laird, G. Landsberg, Z. Mao, M. Narain, S. Piperov, S. Sagir, E. Spencer, R. Syarif

University of California, Davis, Davis, U.S.A.

R. Breedon, D. Burns, M. Calderon De La Barca Sanchez, S. Chauhan, M. Chertok, J. Conway, R. Conway, P.T. Cox, R. Erbacher, C. Flores, G. Funk, M. Gardner, W. Ko, R. Lander, C. Mclean, M. Mulhearn, D. Pellett, J. Pilot, S. Shalhout, M. Shi, J. Smith, M. Squires, D. Stolp, K. Tos, M. Tripathi

University of California, Los Angeles, U.S.A.

M. Bachtis, C. Bravo, R. Cousins, A. Dasgupta, A. Florent, J. Hauser, M. Ignatenko, N. Mccoll, D. Saltzberg, C. Schnaible, V. Valuev, M. Weber

University of California, Riverside, Riverside, U.S.A.

E. Bouvier, K. Burt, R. Clare, J. Ellison, J.W. Gary, S.M.A. Ghiasi Shirazi, G. Hanson, J. Heilman, P. Jandir, E. Kennedy, F. Lacroix, O.R. Long, M. Olmedo Negrete, M.I. Paneva, A. Shrinivas, W. Si, H. Wei, S. Wimpenny, B. R. Yates

University of California, San Diego, La Jolla, U.S.A.

J.G. Branson, G.B. Cerati, S. Cittolin, M. Derdzinski, R. Gerosa, A. Holzner, D. Klein, V. Krutelyov, J. Letts, I. Macneill, D. Olivito, S. Padhi, M. Pieri, M. Sani, V. Sharma, S. Simon, M. Tadel, A. Vartak, S. Wasserbaech ${ }^{68}$, C. Welke, J. Wood, F. Würthwein, A. Yagil, G. Zevi Della Porta

University of California, Santa Barbara - Department of Physics, Santa Barbara, U.S.A.

N. Amin, R. Bhandari, J. Bradmiller-Feld, C. Campagnari, A. Dishaw, V. Dutta, M. Franco Sevilla, C. George, F. Golf, L. Gouskos, J. Gran, R. Heller, J. Incandela, S.D. Mullin, A. Ovcharova, H. Qu, J. Richman, D. Stuart, I. Suarez, J. Yoo 


\section{California Institute of Technology, Pasadena, U.S.A.}

D. Anderson, J. Bendavid, A. Bornheim, J. Bunn, J. Duarte, J.M. Lawhorn, A. Mott, H.B. Newman, C. Pena, M. Spiropulu, J.R. Vlimant, S. Xie, R.Y. Zhu

Carnegie Mellon University, Pittsburgh, U.S.A.

M.B. Andrews, T. Ferguson, M. Paulini, J. Russ, M. Sun, H. Vogel, I. Vorobiev, M. Weinberg

\section{University of Colorado Boulder, Boulder, U.S.A.}

J.P. Cumalat, W.T. Ford, F. Jensen, A. Johnson, M. Krohn, S. Leontsinis, T. Mulholland, K. Stenson, S.R. Wagner

\section{Cornell University, Ithaca, U.S.A.}

J. Alexander, J. Chaves, J. Chu, S. Dittmer, K. Mcdermott, N. Mirman, G. Nicolas Kaufman, J.R. Patterson, A. Rinkevicius, A. Ryd, L. Skinnari, L. Soffi, S.M. Tan, Z. Tao, J. Thom, J. Tucker, P. Wittich, M. Zientek

Fairfield University, Fairfield, U.S.A.

D. Winn

Fermi National Accelerator Laboratory, Batavia, U.S.A.

S. Abdullin, M. Albrow, G. Apollinari, A. Apresyan, S. Banerjee, L.A.T. Bauerdick, A. Beretvas, J. Berryhill, P.C. Bhat, G. Bolla, K. Burkett, J.N. Butler, H.W.K. Cheung, F. Chlebana, S. Cihangir ${ }^{\dagger}$, M. Cremonesi, V.D. Elvira, I. Fisk, J. Freeman, E. Gottschalk, L. Gray, D. Green, S. Grünendahl, O. Gutsche, D. Hare, R.M. Harris, S. Hasegawa, J. Hirschauer, Z. Hu, B. Jayatilaka, S. Jindariani, M. Johnson, U. Joshi, B. Klima, B. Kreis, S. Lammel, J. Linacre, D. Lincoln, R. Lipton, M. Liu, T. Liu, R. Lopes De Sá, J. Lykken, K. Maeshima, N. Magini, J.M. Marraffino, S. Maruyama, D. Mason, P. McBride, P. Merkel, S. Mrenna, S. Nahn, V. O’Dell, K. Pedro, O. Prokofyev, G. Rakness, L. Ristori, E. SextonKennedy, A. Soha, W.J. Spalding, L. Spiegel, S. Stoynev, J. Strait, N. Strobbe, L. Taylor, S. Tkaczyk, N.V. Tran, L. Uplegger, E.W. Vaandering, C. Vernieri, M. Verzocchi, R. Vidal, M. Wang, H.A. Weber, A. Whitbeck, Y. Wu

University of Florida, Gainesville, U.S.A.

D. Acosta, P. Avery, P. Bortignon, D. Bourilkov, A. Brinkerhoff, A. Carnes, M. Carver, D. Curry, S. Das, R.D. Field, I.K. Furic, J. Konigsberg, A. Korytov, J.F. Low, P. Ma, K. Matchev, H. Mei, G. Mitselmakher, D. Rank, L. Shchutska, D. Sperka, L. Thomas, J. Wang, S. Wang, J. Yelton

Florida International University, Miami, U.S.A.

S. Linn, P. Markowitz, G. Martinez, J.L. Rodriguez

Florida State University, Tallahassee, U.S.A.

A. Ackert, T. Adams, A. Askew, S. Bein, S. Hagopian, V. Hagopian, K.F. Johnson, T. Kolberg, T. Perry, H. Prosper, A. Santra, R. Yohay 
Florida Institute of Technology, Melbourne, U.S.A.

M.M. Baarmand, V. Bhopatkar, S. Colafranceschi, M. Hohlmann, D. Noonan, T. Roy, F. Yumiceva

University of Illinois at Chicago (UIC), Chicago, U.S.A.

M.R. Adams, L. Apanasevich, D. Berry, R.R. Betts, I. Bucinskaite, R. Cavanaugh, X. Chen, O. Evdokimov, L. Gauthier, C.E. Gerber, D.A. Hangal, D.J. Hofman, K. Jung, J. Kamin, I.D. Sandoval Gonzalez, H. Trauger, N. Varelas, H. Wang, Z. Wu, M. Zakaria, J. Zhang

The University of Iowa, Iowa City, U.S.A.

B. Bilki ${ }^{69}$, W. Clarida, K. Dilsiz, S. Durgut, R.P. Gandrajula, M. Haytmyradov, V. Khristenko, J.-P. Merlo, H. Mermerkaya ${ }^{70}$, A. Mestvirishvili, A. Moeller, J. Nachtman, H. Ogul, Y. Onel, F. Ozok ${ }^{71}$, A. Penzo, C. Snyder, E. Tiras, J. Wetzel, K. Yi

Johns Hopkins University, Baltimore, U.S.A.

B. Blumenfeld, A. Cocoros, N. Eminizer, D. Fehling, L. Feng, A.V. Gritsan, P. Maksimovic, J. Roskes, U. Sarica, M. Swartz, M. Xiao, C. You

The University of Kansas, Lawrence, U.S.A.

A. Al-bataineh, P. Baringer, A. Bean, S. Boren, J. Bowen, J. Castle, L. Forthomme,

S. Khalil, A. Kropivnitskaya, D. Majumder, W. Mcbrayer, M. Murray, S. Sanders, R. Stringer, J.D. Tapia Takaki, Q. Wang

Kansas State University, Manhattan, U.S.A.

A. Ivanov, K. Kaadze, Y. Maravin, A. Mohammadi, L.K. Saini, N. Skhirtladze, S. Toda

Lawrence Livermore National Laboratory, Livermore, U.S.A.

F. Rebassoo, D. Wright

University of Maryland, College Park, U.S.A.

C. Anelli, A. Baden, O. Baron, A. Belloni, B. Calvert, S.C. Eno, C. Ferraioli, J.A. Gomez, N.J. Hadley, S. Jabeen, G.Y. Jeng, R.G. Kellogg, J. Kunkle, A.C. Mignerey, F. Ricci-Tam, Y.H. Shin, A. Skuja, M.B. Tonjes, S.C. Tonwar

Massachusetts Institute of Technology, Cambridge, U.S.A.

D. Abercrombie, B. Allen, A. Apyan, V. Azzolini, R. Barbieri, A. Baty, R. Bi, K. Bierwagen, S. Brandt, W. Busza, I.A. Cali, M. D’Alfonso, Z. Demiragli, G. Gomez Ceballos, M. Goncharov, D. Hsu, Y. Iiyama, G.M. Innocenti, M. Klute, D. Kovalskyi, K. Krajczar, Y.S. Lai, Y.-J. Lee, A. Levin, P.D. Luckey, B. Maier, A.C. Marini, C. Mcginn, C. Mironov, S. Narayanan, X. Niu, C. Paus, C. Roland, G. Roland, J. Salfeld-Nebgen, G.S.F. Stephans, K. Tatar, D. Velicanu, J. Wang, T.W. Wang, B. Wyslouch

University of Minnesota, Minneapolis, U.S.A.

A.C. Benvenuti, R.M. Chatterjee, A. Evans, P. Hansen, S. Kalafut, S.C. Kao, Y. Kubota, Z. Lesko, J. Mans, S. Nourbakhsh, N. Ruckstuhl, R. Rusack, N. Tambe, J. Turkewitz

University of Mississippi, Oxford, U.S.A.

J.G. Acosta, S. Oliveros 
University of Nebraska-Lincoln, Lincoln, U.S.A.

E. Avdeeva, K. Bloom, D.R. Claes, C. Fangmeier, R. Gonzalez Suarez, R. Kamalieddin, I. Kravchenko, A. Malta Rodrigues, J. Monroy, J.E. Siado, G.R. Snow, B. Stieger

State University of New York at Buffalo, Buffalo, U.S.A.

M. Alyari, J. Dolen, A. Godshalk, C. Harrington, I. Iashvili, J. Kaisen, D. Nguyen, A. Parker, S. Rappoccio, B. Roozbahani

Northeastern University, Boston, U.S.A.

G. Alverson, E. Barberis, A. Hortiangtham, A. Massironi, D.M. Morse, D. Nash, T. Orimoto, R. Teixeira De Lima, D. Trocino, R.-J. Wang, D. Wood

Northwestern University, Evanston, U.S.A.

S. Bhattacharya, O. Charaf, K.A. Hahn, A. Kumar, N. Mucia, N. Odell, B. Pollack, M.H. Schmitt, K. Sung, M. Trovato, M. Velasco

\section{University of Notre Dame, Notre Dame, U.S.A.}

N. Dev, M. Hildreth, K. Hurtado Anampa, C. Jessop, D.J. Karmgard, N. Kellams, K. Lannon, N. Marinelli, F. Meng, C. Mueller, Y. Musienko ${ }^{37}$, M. Planer, A. Reinsvold, R. Ruchti, N. Rupprecht, G. Smith, S. Taroni, M. Wayne, M. Wolf, A. Woodard

The Ohio State University, Columbus, U.S.A.

J. Alimena, L. Antonelli, B. Bylsma, L.S. Durkin, S. Flowers, B. Francis, A. Hart, C. Hill, W. Ji, B. Liu, W. Luo, D. Puigh, B.L. Winer, H.W. Wulsin

Princeton University, Princeton, U.S.A.

S. Cooperstein, O. Driga, P. Elmer, J. Hardenbrook, P. Hebda, D. Lange, J. Luo, D. Marlow, T. Medvedeva, K. Mei, I. Ojalvo, J. Olsen, C. Palmer, P. Piroué, D. Stickland, A. Svyatkovskiy, C. Tully

University of Puerto Rico, Mayaguez, U.S.A.

S. Malik

Purdue University, West Lafayette, U.S.A.

A. Barker, V.E. Barnes, S. Folgueras, L. Gutay, M.K. Jha, M. Jones, A.W. Jung, A. Khatiwada, D.H. Miller, N. Neumeister, J.F. Schulte, X. Shi, J. Sun, F. Wang, W. Xie Purdue University Northwest, Hammond, U.S.A.

N. Parashar, J. Stupak

Rice University, Houston, U.S.A.

A. Adair, B. Akgun, Z. Chen, K.M. Ecklund, F.J.M. Geurts, M. Guilbaud, W. Li, B. Michlin, M. Northup, B.P. Padley, J. Roberts, J. Rorie, Z. Tu, J. Zabel

University of Rochester, Rochester, U.S.A.

B. Betchart, A. Bodek, P. de Barbaro, R. Demina, Y.t. Duh, T. Ferbel, M. Galanti, A. Garcia-Bellido, J. Han, O. Hindrichs, A. Khukhunaishvili, K.H. Lo, P. Tan, M. Verzetti 
Rutgers, The State University of New Jersey, Piscataway, U.S.A.

A. Agapitos, J.P. Chou, Y. Gershtein, T.A. Gómez Espinosa, E. Halkiadakis, M. Heindl,

E. Hughes, S. Kaplan, R. Kunnawalkam Elayavalli, S. Kyriacou, A. Lath, R. Montalvo, K. Nash, M. Osherson, H. Saka, S. Salur, S. Schnetzer, D. Sheffield, S. Somalwar, R. Stone, S. Thomas, P. Thomassen, M. Walker

University of Tennessee, Knoxville, U.S.A.

A.G. Delannoy, M. Foerster, J. Heideman, G. Riley, K. Rose, S. Spanier, K. Thapa

Texas A\&M University, College Station, U.S.A.

O. Bouhali ${ }^{72}$, A. Celik, M. Dalchenko, M. De Mattia, A. Delgado, S. Dildick, R. Eusebi, J. Gilmore, T. Huang, E. Juska, T. Kamon ${ }^{73}$, R. Mueller, Y. Pakhotin, R. Patel, A. Perloff, L. Perniè, D. Rathjens, A. Safonov, A. Tatarinov, K.A. Ulmer

Texas Tech University, Lubbock, U.S.A.

N. Akchurin, J. Damgov, F. De Guio, C. Dragoiu, P.R. Dudero, J. Faulkner, E. Gurpinar, S. Kunori, K. Lamichhane, S.W. Lee, T. Libeiro, T. Peltola, S. Undleeb, I. Volobouev, Z. Wang

\section{Vanderbilt University, Nashville, U.S.A.}

S. Greene, A. Gurrola, R. Janjam, W. Johns, C. Maguire, A. Melo, H. Ni, P. Sheldon, S. Tuo, J. Velkovska, Q. Xu

\section{University of Virginia, Charlottesville, U.S.A.}

M.W. Arenton, P. Barria, B. Cox, J. Goodell, R. Hirosky, A. Ledovskoy, H. Li, C. Neu, T. Sinthuprasith, X. Sun, Y. Wang, E. Wolfe, F. Xia

Wayne State University, Detroit, U.S.A.

C. Clarke, R. Harr, P.E. Karchin, J. Sturdy, S. Zaleski

University of Wisconsin - Madison, Madison, WI, U.S.A.

D.A. Belknap, J. Buchanan, C. Caillol, S. Dasu, L. Dodd, S. Duric, B. Gomber, M. Grothe, M. Herndon, A. Hervé, U. Hussain, P. Klabbers, A. Lanaro, A. Levine, K. Long, R. Loveless, G.A. Pierro, G. Polese, T. Ruggles, A. Savin, N. Smith, W.H. Smith, D. Taylor, N. Woods

$\dagger$ : Deceased

1: Also at Vienna University of Technology, Vienna, Austria

2: Also at State Key Laboratory of Nuclear Physics and Technology, Peking University, Beijing, China

3: Also at Institut Pluridisciplinaire Hubert Curien (IPHC), Université de Strasbourg, CNRS/IN2P3, Strasbourg, France

4: Also at Universidade Estadual de Campinas, Campinas, Brazil

5: Also at Universidade Federal de Pelotas, Pelotas, Brazil

6: Also at Université Libre de Bruxelles, Bruxelles, Belgium

7: Also at Deutsches Elektronen-Synchrotron, Hamburg, Germany

8: Also at Universidad de Antioquia, Medellin, Colombia 
9: Also at Joint Institute for Nuclear Research, Dubna, Russia

10: Now at Cairo University, Cairo, Egypt

11: Also at Fayoum University, El-Fayoum, Egypt

12: Now at British University in Egypt, Cairo, Egypt

13: Now at Ain Shams University, Cairo, Egypt

14: Also at Université de Haute Alsace, Mulhouse, France

15: Also at Skobeltsyn Institute of Nuclear Physics, Lomonosov Moscow State University, Moscow, Russia

16: Also at CERN, European Organization for Nuclear Research, Geneva, Switzerland

17: Also at RWTH Aachen University, III. Physikalisches Institut A, Aachen, Germany

18: Also at University of Hamburg, Hamburg, Germany

19: Also at Brandenburg University of Technology, Cottbus, Germany

20: Also at Institute of Nuclear Research ATOMKI, Debrecen, Hungary

21: Also at MTA-ELTE Lendület CMS Particle and Nuclear Physics Group, Eötvös Loránd University, Budapest, Hungary

22: Also at Institute of Physics, University of Debrecen, Debrecen, Hungary

23: Also at Indian Institute of Technology Bhubaneswar, Bhubaneswar, India

24: Also at University of Visva-Bharati, Santiniketan, India

25: Also at Indian Institute of Science Education and Research, Bhopal, India

26: Also at Institute of Physics, Bhubaneswar, India

27: Also at University of Ruhuna, Matara, Sri Lanka

28: Also at Isfahan University of Technology, Isfahan, Iran

29: Also at Yazd University, Yazd, Iran

30: Also at Plasma Physics Research Center, Science and Research Branch, Islamic Azad University, Tehran, Iran

31: Also at Università degli Studi di Siena, Siena, Italy

32: Also at Purdue University, West Lafayette, U.S.A.

33: Also at International Islamic University of Malaysia, Kuala Lumpur, Malaysia

34: Also at Malaysian Nuclear Agency, MOSTI, Kajang, Malaysia

35: Also at Consejo Nacional de Ciencia y Tecnología, Mexico city, Mexico

36: Also at Warsaw University of Technology, Institute of Electronic Systems, Warsaw, Poland

37: Also at Institute for Nuclear Research, Moscow, Russia

38: Now at National Research Nuclear University 'Moscow Engineering Physics Institute' (MEPhI), Moscow, Russia

39: Also at St. Petersburg State Polytechnical University, St. Petersburg, Russia

40: Also at University of Florida, Gainesville, U.S.A.

41: Also at P.N. Lebedev Physical Institute, Moscow, Russia

42: Also at California Institute of Technology, Pasadena, U.S.A.

43: Also at Budker Institute of Nuclear Physics, Novosibirsk, Russia

44: Also at Faculty of Physics, University of Belgrade, Belgrade, Serbia

45: Also at INFN Sezione di Roma; Università di Roma, Roma, Italy

46: Also at University of Belgrade, Faculty of Physics and Vinca Institute of Nuclear Sciences, Belgrade, Serbia

47: Also at Scuola Normale e Sezione dell'INFN, Pisa, Italy

48: Also at National and Kapodistrian University of Athens, Athens, Greece

49: Also at Riga Technical University, Riga, Latvia

50: Also at Institute for Theoretical and Experimental Physics, Moscow, Russia

51: Also at Albert Einstein Center for Fundamental Physics, Bern, Switzerland 
52: Also at Gaziosmanpasa University, Tokat, Turkey

53: Also at Istanbul Aydin University, Istanbul, Turkey

54: Also at Mersin University, Mersin, Turkey

55: Also at Cag University, Mersin, Turkey

56: Also at Piri Reis University, Istanbul, Turkey

57: Also at Adiyaman University, Adiyaman, Turkey

58: Also at Ozyegin University, Istanbul, Turkey

59: Also at Izmir Institute of Technology, Izmir, Turkey

60: Also at Marmara University, Istanbul, Turkey

61: Also at Kafkas University, Kars, Turkey

62: Also at Istanbul Bilgi University, Istanbul, Turkey

63: Also at Yildiz Technical University, Istanbul, Turkey

64: Also at Hacettepe University, Ankara, Turkey

65: Also at Rutherford Appleton Laboratory, Didcot, United Kingdom

66: Also at School of Physics and Astronomy, University of Southampton, Southampton, United Kingdom

67: Also at Instituto de Astrofísica de Canarias, La Laguna, Spain

68: Also at Utah Valley University, Orem, U.S.A.

69: Also at Argonne National Laboratory, Argonne, U.S.A.

70: Also at Erzincan University, Erzincan, Turkey

71: Also at Mimar Sinan University, Istanbul, Istanbul, Turkey

72: Also at Texas A\&M University at Qatar, Doha, Qatar

73: Also at Kyungpook National University, Daegu, Korea 